\title{
Understanding Internal Chirality Induction of Triarylsilyl Ethers Formed From Enantiopure Alcohols
}

Li Wang, Tian Zhang, Brandon K. Redden, Cody I. Sheppard, Robert W. Clark, Mark D.

Smith, Sheryl L. Wiskur*

University of South Carolina, 631 Sumter St., Columbia, SC 29208

Supporting Information

S1. Table of Contents

S2. CD Spectra of Triarylsilyl Ether Derivatives

S17. Crystal Structure Information

S23. Molecular Modeling Information

S33. References

S34. NMR Spectroscopic Data of Selected Compounds 
CD Spectra of Triarylsilyl Ether Derivatives.
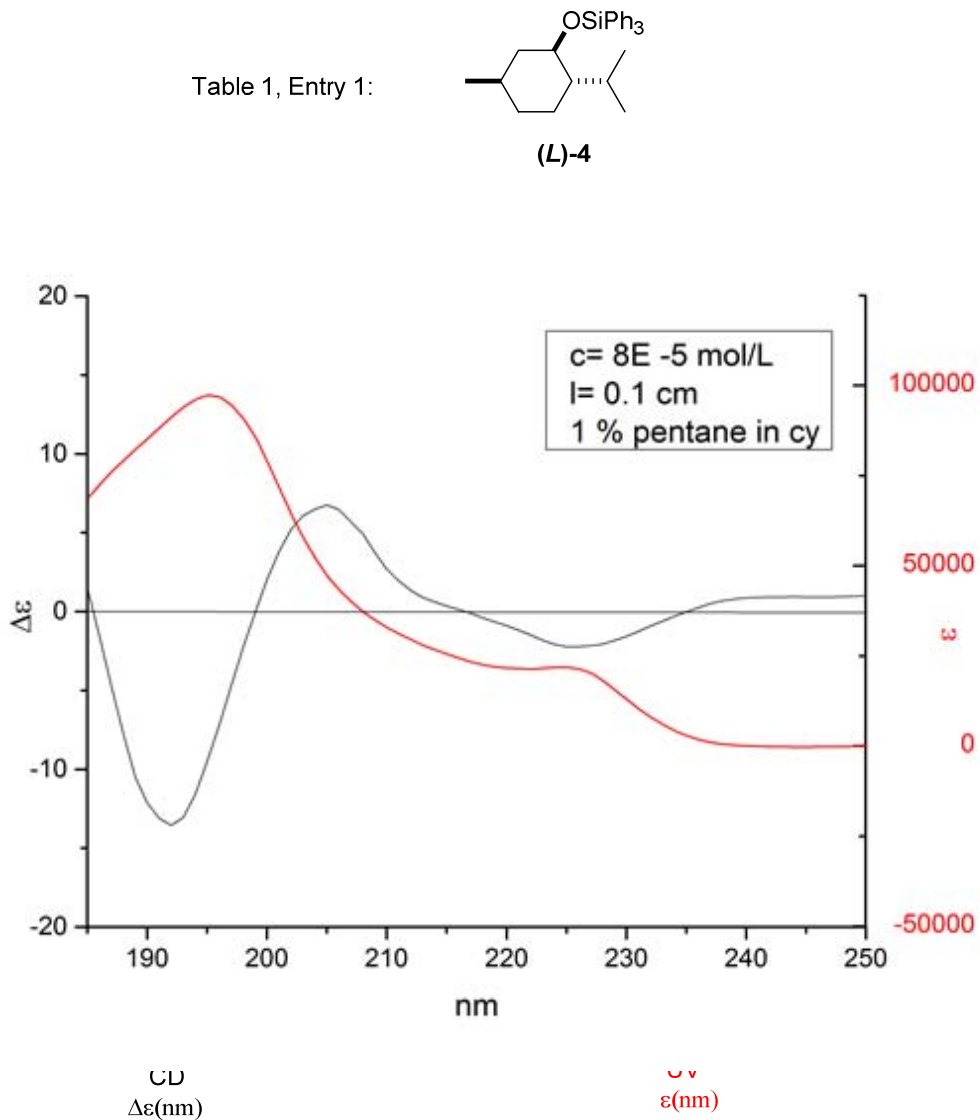
6.7
205
$-13.4$
192
97361
195 

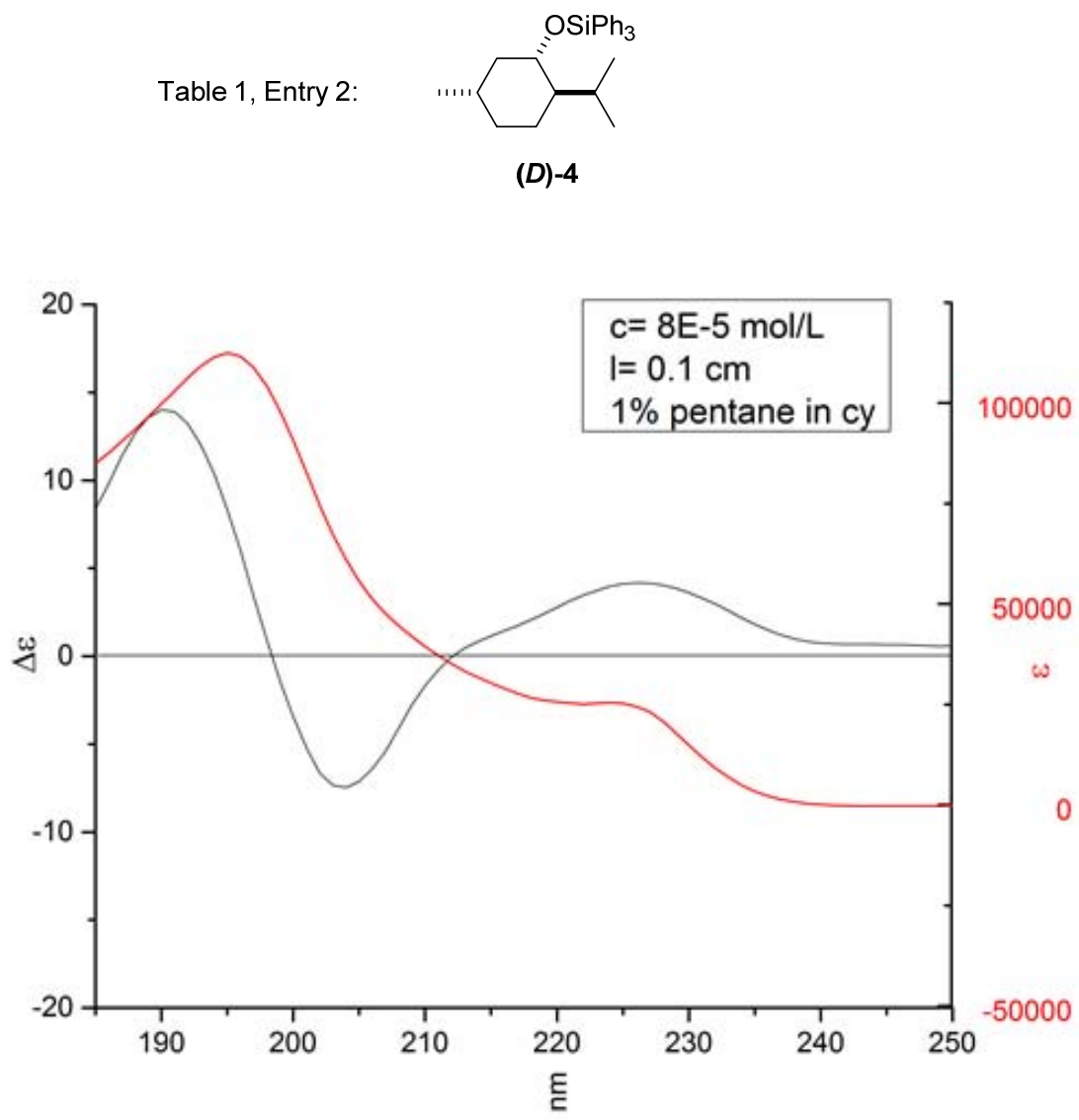

$\Delta \varepsilon(\mathrm{nm})$

$\varepsilon(\mathrm{nm})$

$\begin{array}{cccc}-7.5 & 204 & 112490 & 195 \\ 14 & 190 & 190\end{array}$


Table 1, Entry 3:
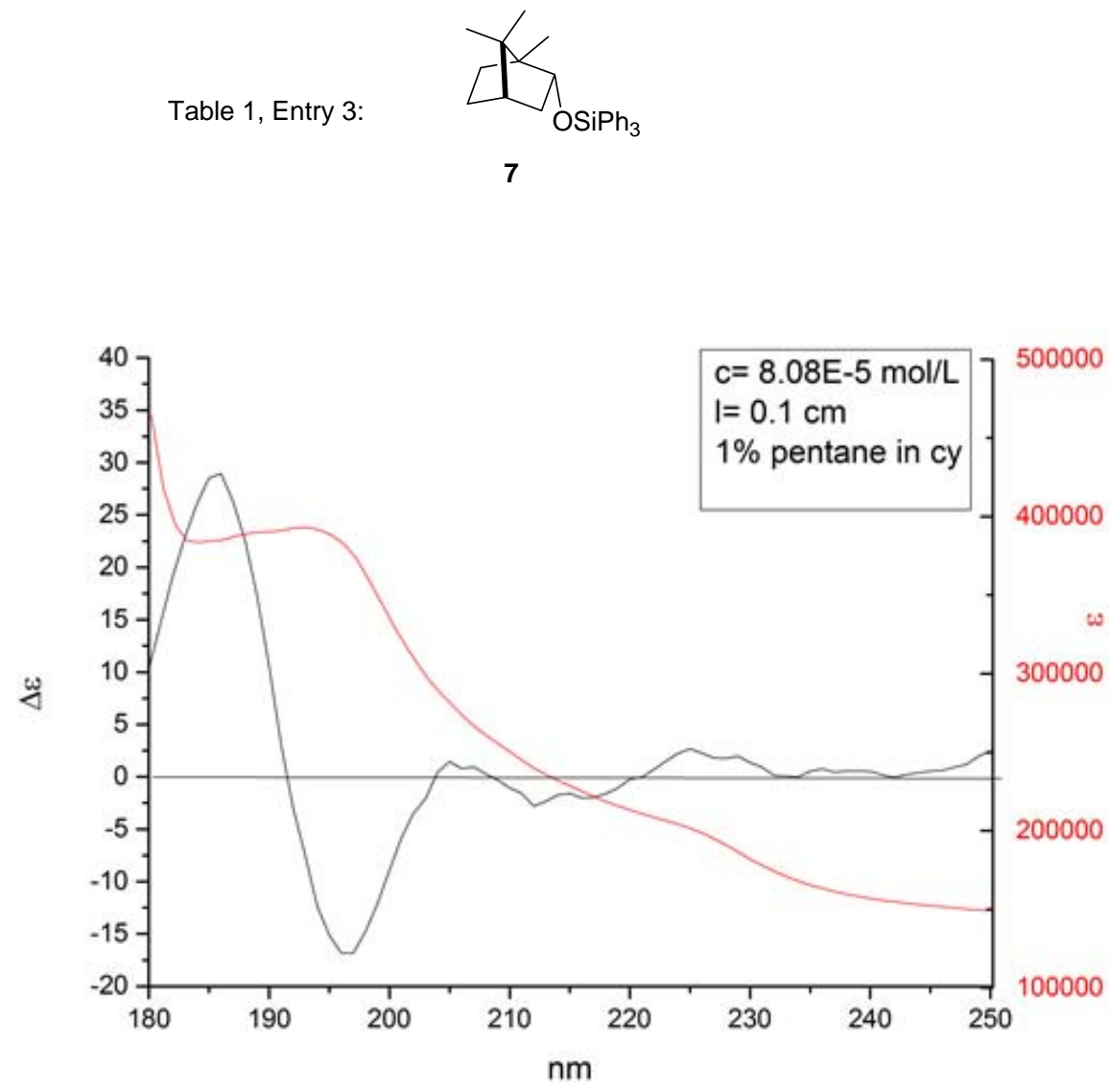

CD

$\Delta \varepsilon(\mathrm{nm})$
UV

$\varepsilon(\mathrm{nm})$

$-17$

29

196
186

393104

193 
Table 1, Entry 4:

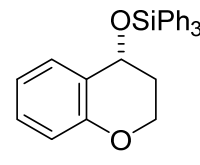

(R)-5

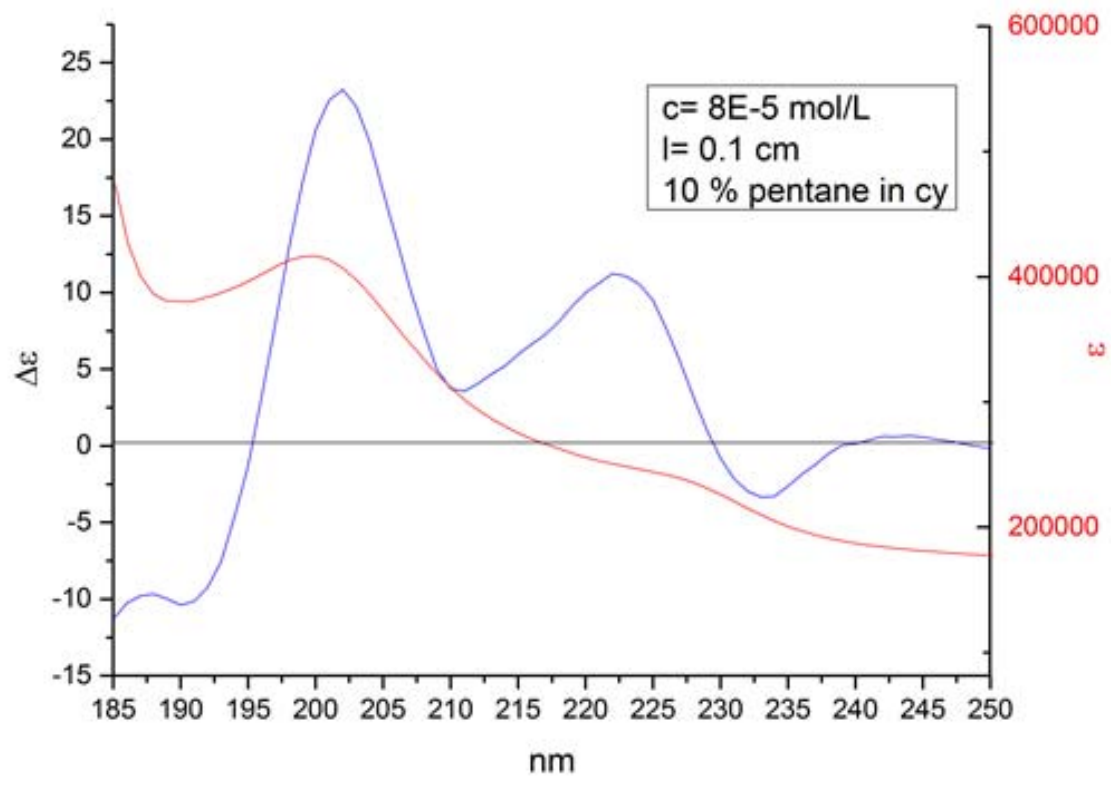

CD

$\Delta \varepsilon(\mathrm{nm})$
UV

$\varepsilon(\mathrm{nm})$ 

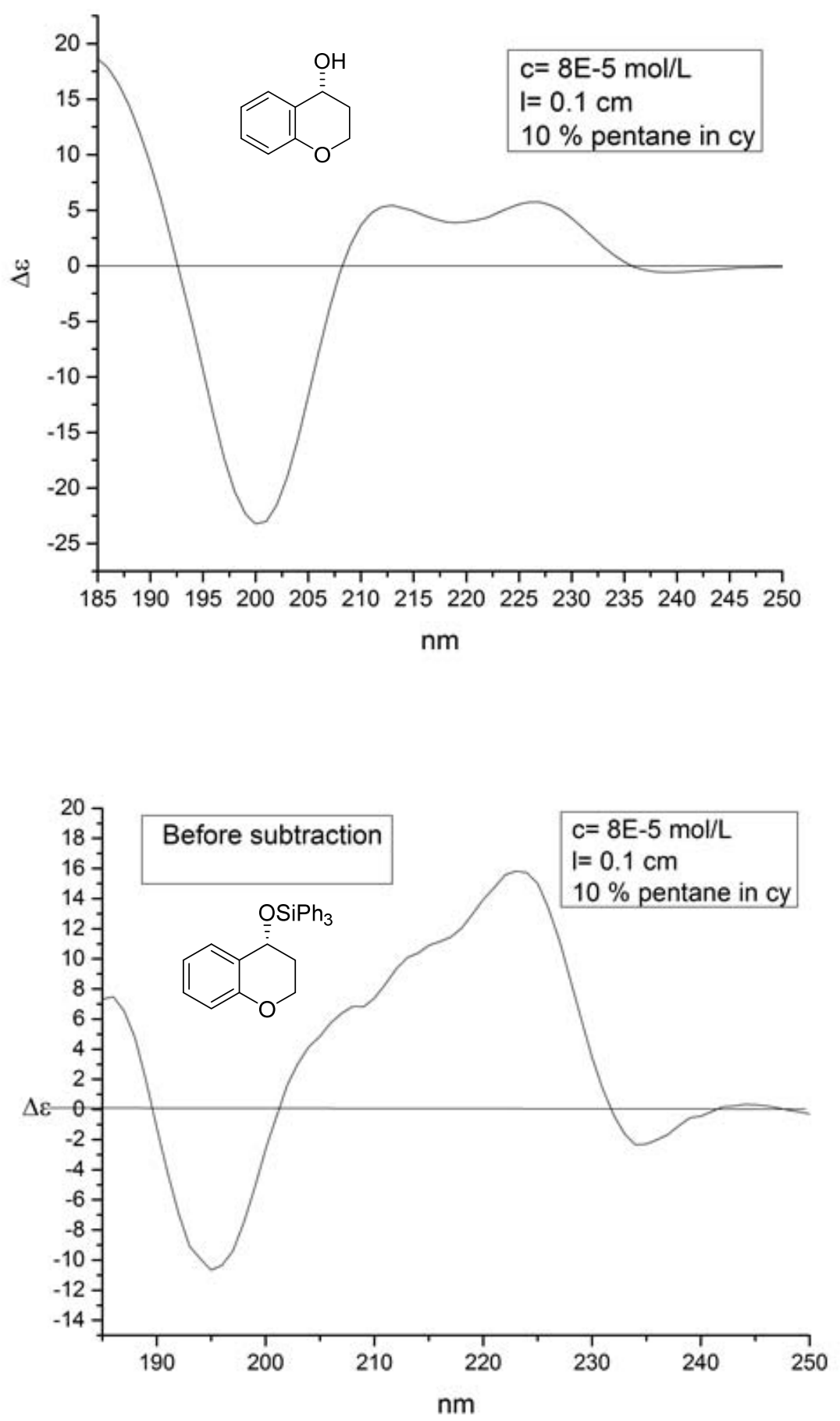
Table 1, Entry 5:

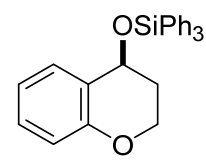

(S)-5

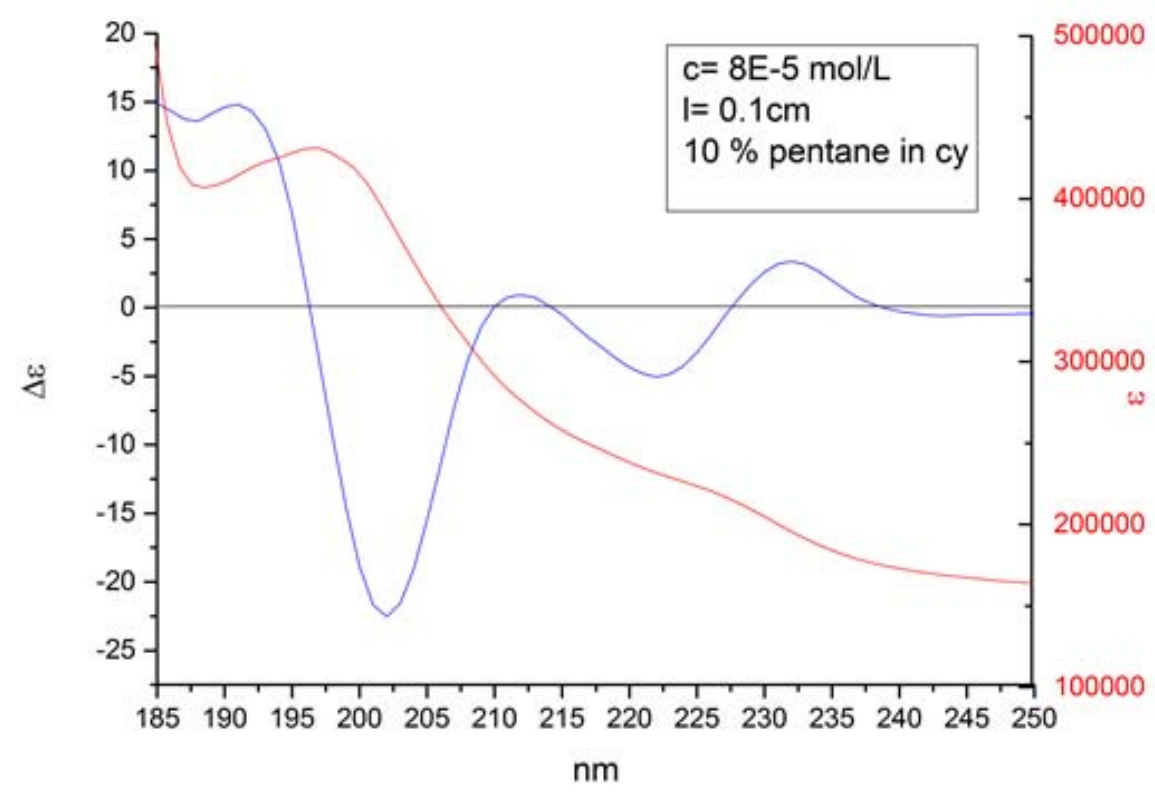

CD

$\Delta \varepsilon(\mathrm{nm})$
UV

$\varepsilon(\mathrm{nm})$

431104

193 

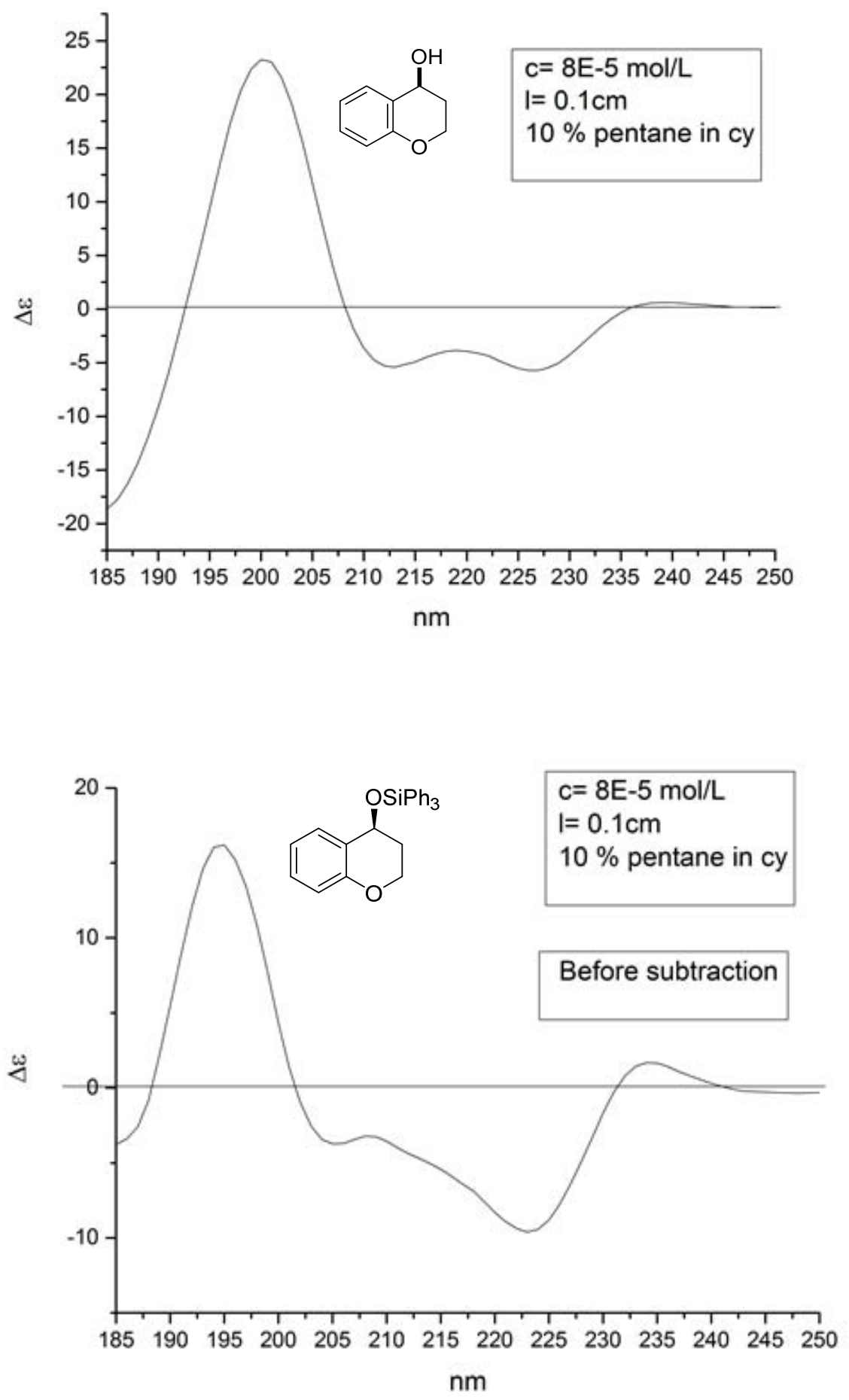
Table 1, Entry 6:
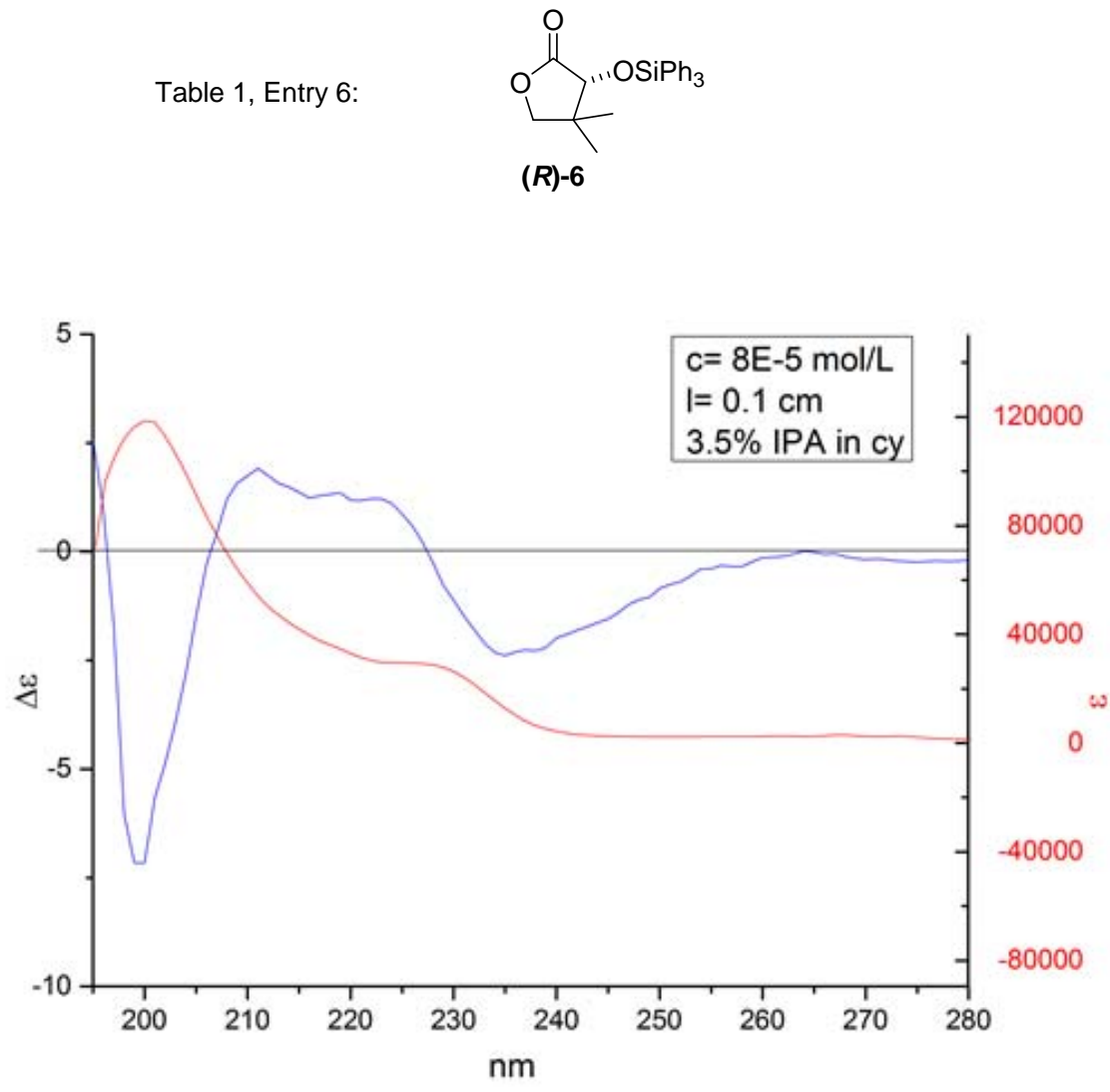

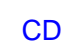

$\Delta \varepsilon(\mathrm{nm})$
UV

$\varepsilon(\mathrm{nm})$

$\begin{array}{rrrr}1.9 & 210 & 118443 & 195 \\ -7.2 & 199 & & \end{array}$



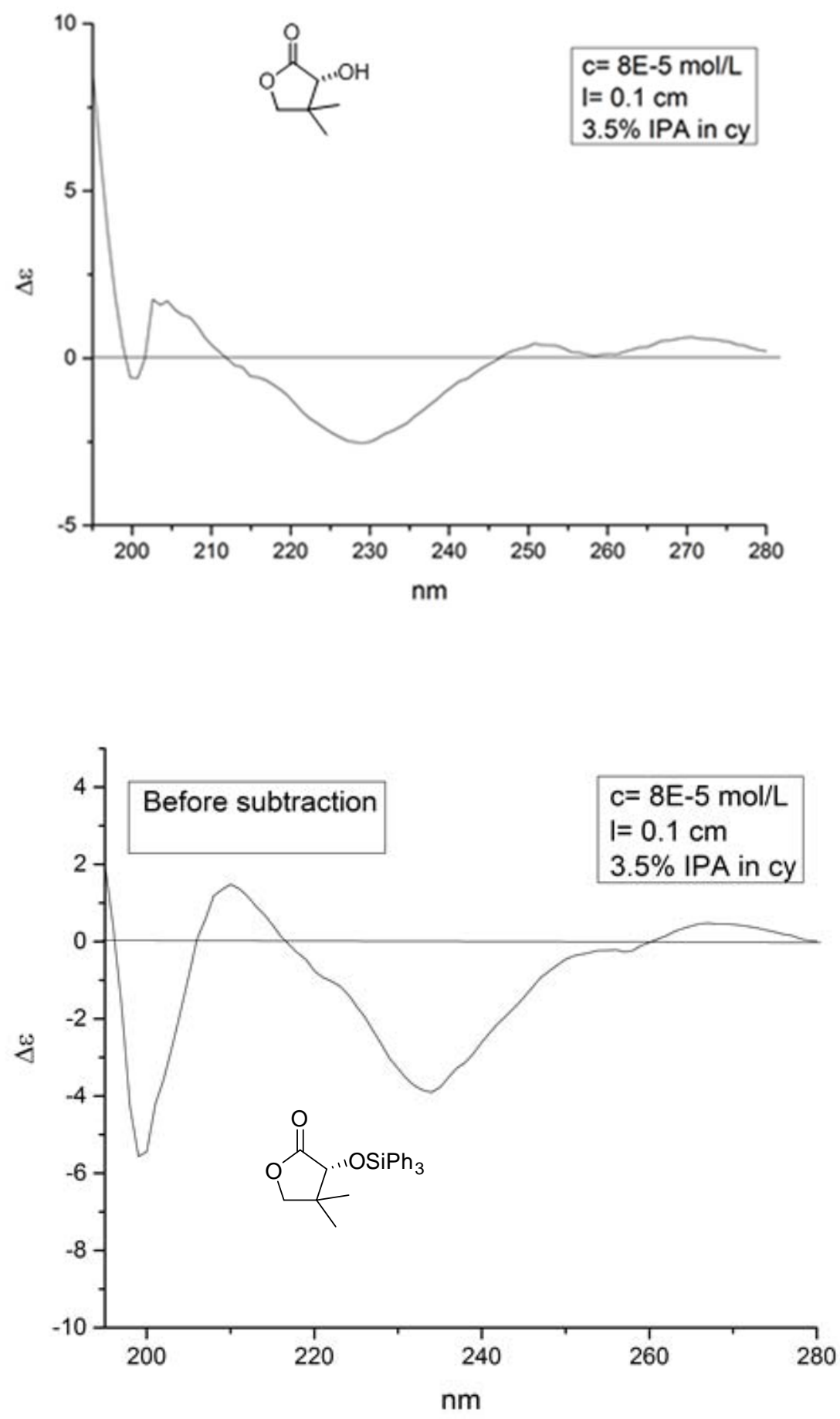

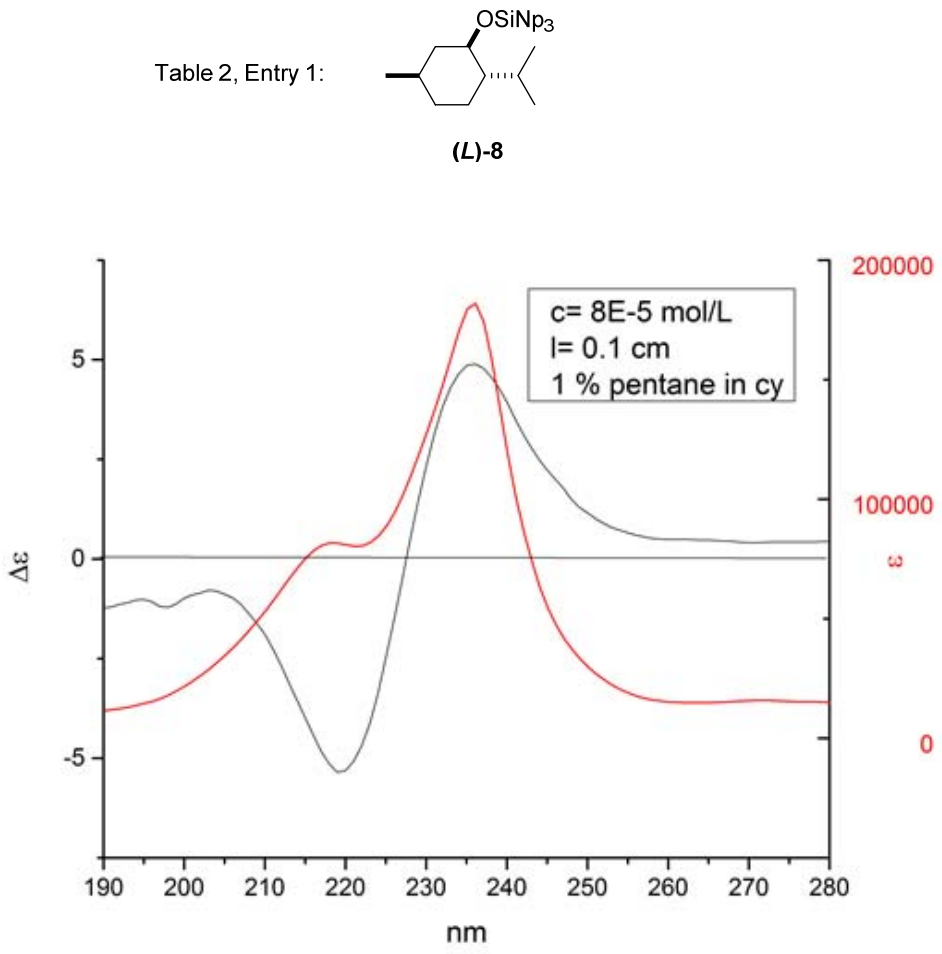

CD

$\Delta \varepsilon(\mathrm{nm})$
UV

$\varepsilon(\mathrm{nm})$
4.9

$-5.3$ 

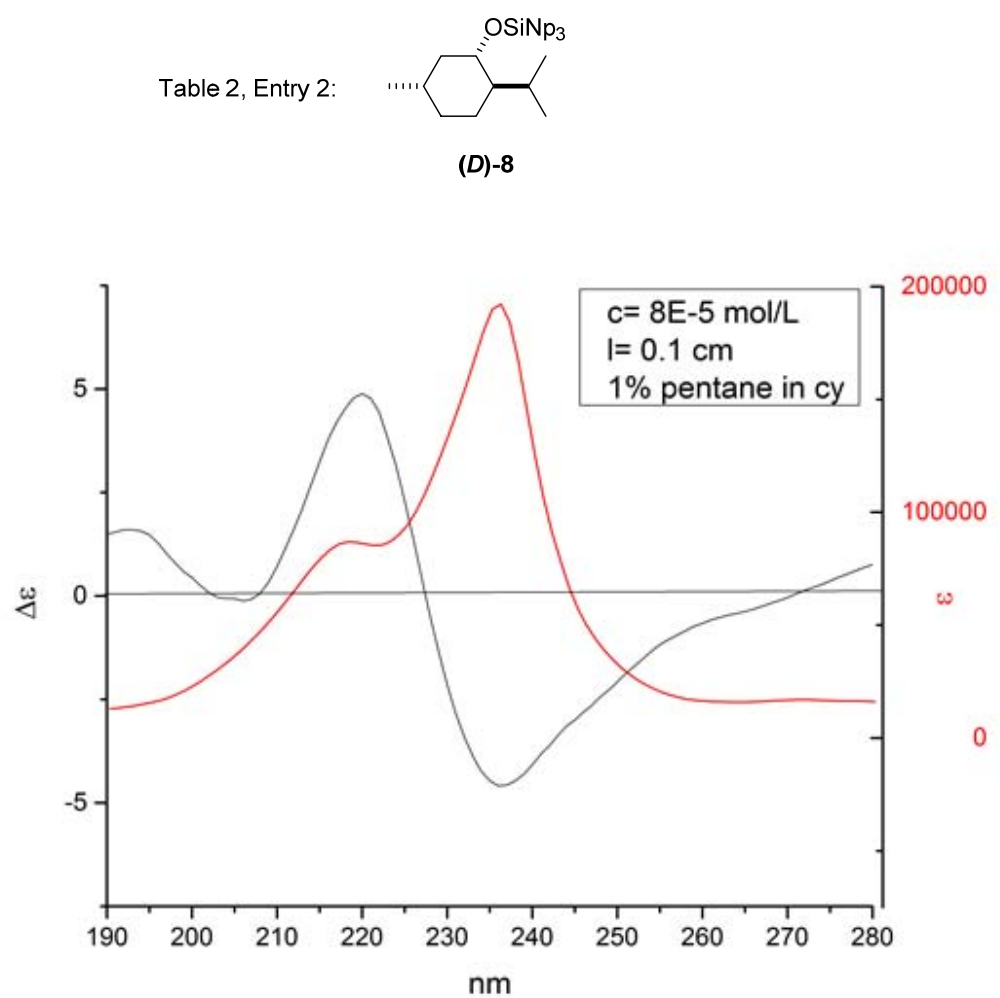

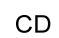

$\Delta \varepsilon(\mathrm{nm})$

192216

236 

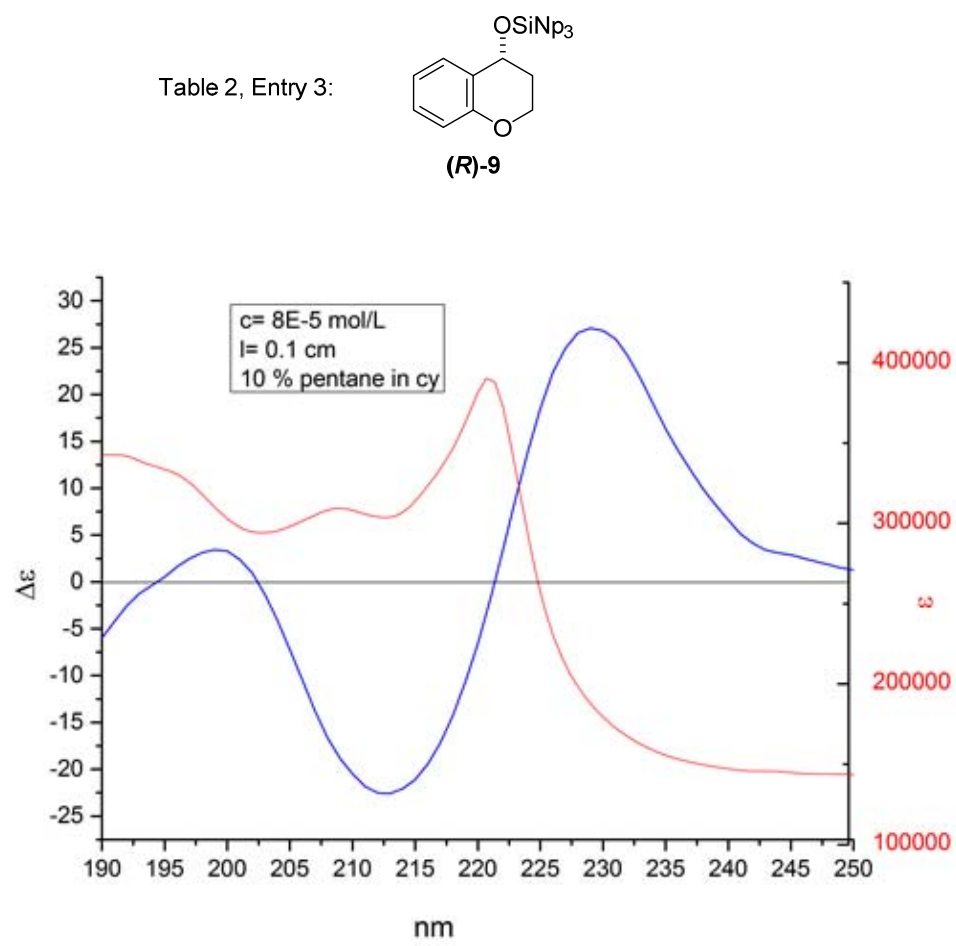

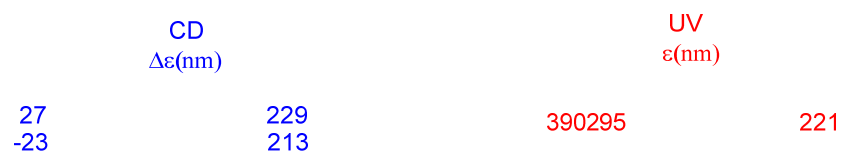



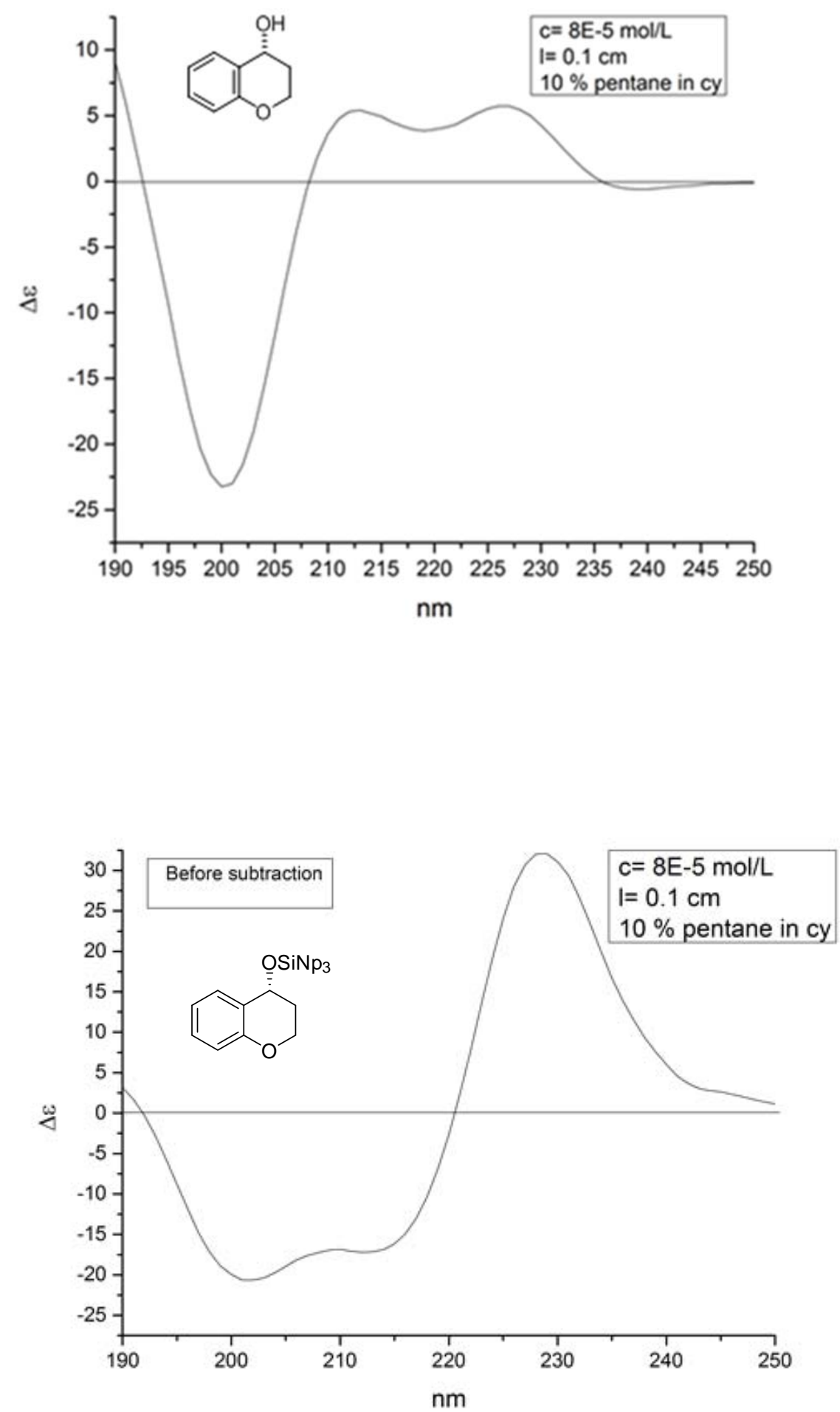


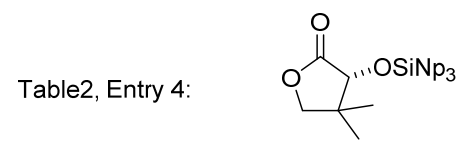

(R)-10
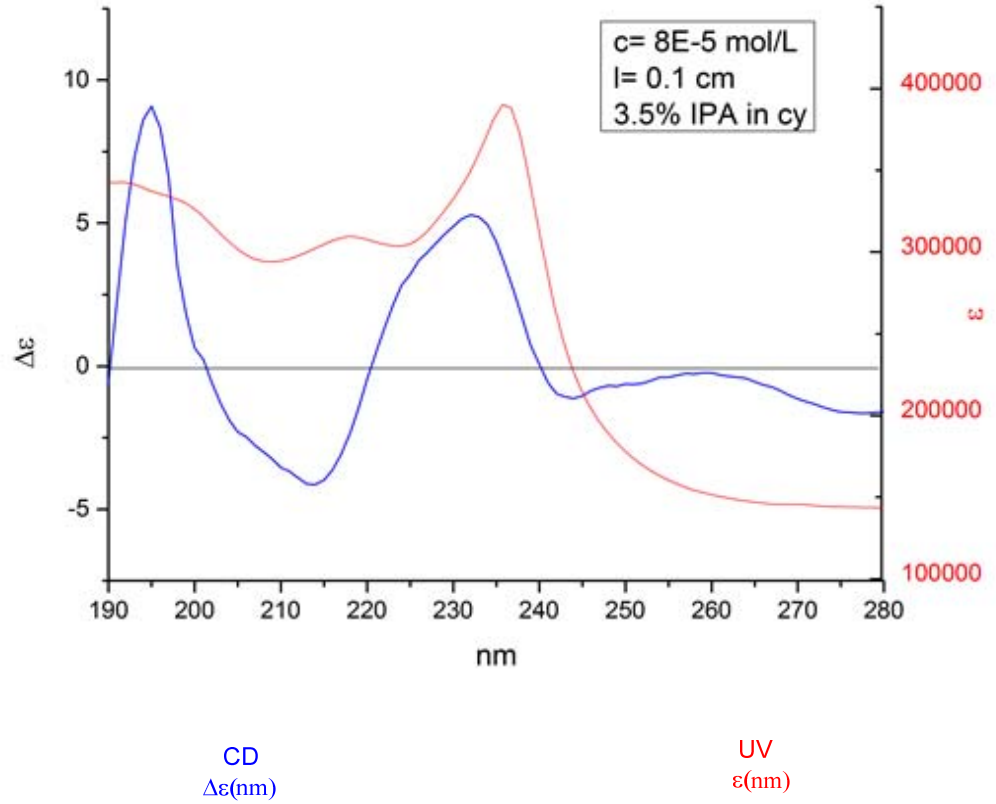

$\begin{array}{rrrr}5.3 & 232 & 390295 & 236 \\ -4.1 & 214 & \end{array}$



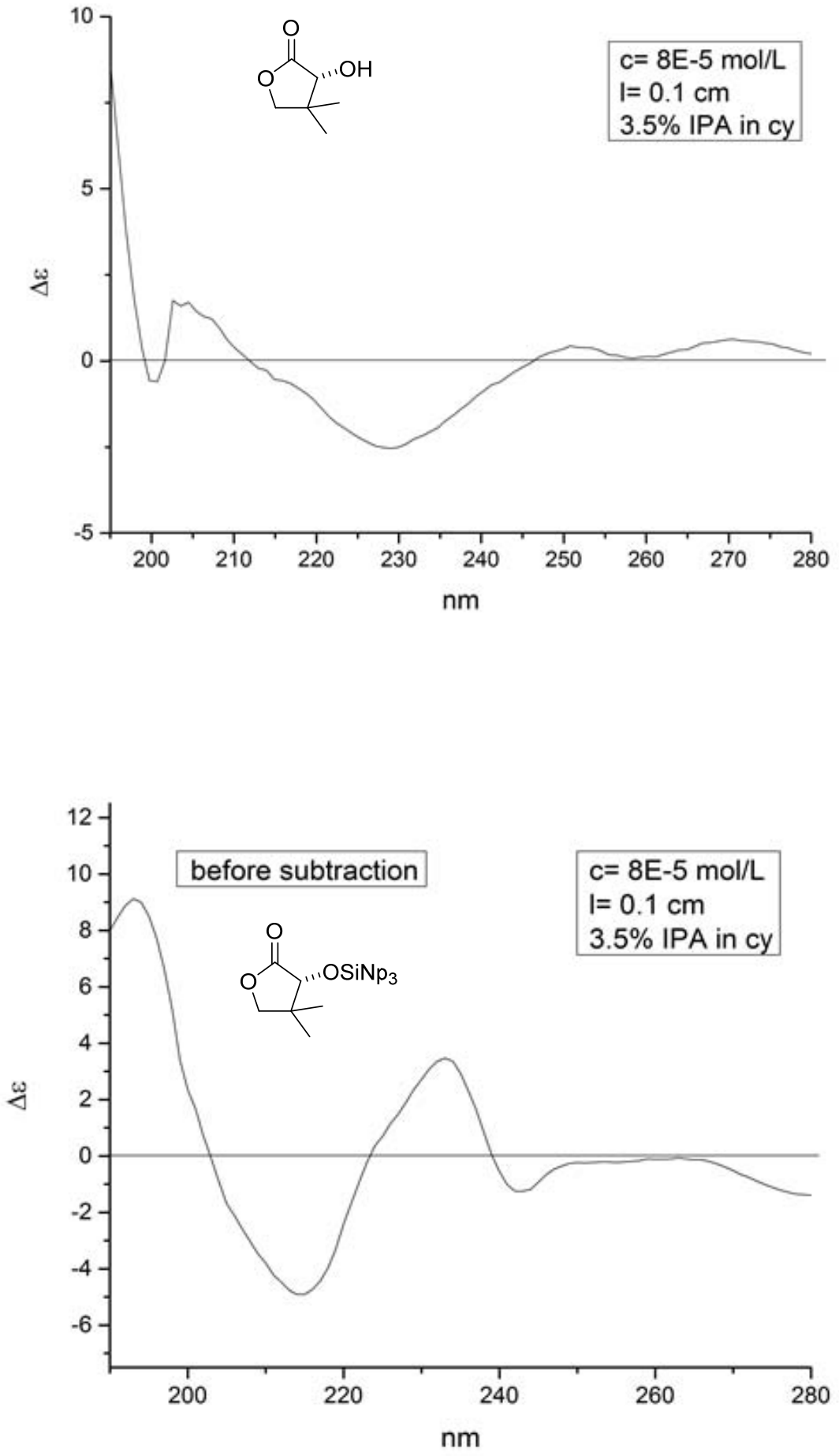


\section{Crystal Structure Information}

X-Ray Structure Determination of (S)-5. X-ray intensity data from a colorless plate crystal were collected at 296(2) K using a diffractometer (Mo Ka radiation, I $=0.71073 \AA$ ). ${ }^{1}$ The raw area detector data frames were reduced with the Bruker SAINT+ program ${ }^{1}$. Final unit cell parameters were determined by least-squares refinement of 2025 reflections from the data set. Direct methods structure solution, difference Fourier calculations and full-matrix least-squares refinement against $F^{2}$ were performed with SHELXS $/ L^{2}$ as implemented in OLEX ${ }^{3}$.

The compound crystallizes in the monoclinic system. Intensity statistics indicated an acentric structure (mean $\left|E^{*} E-1\right|=0.722$ ). The space group $P 2_{1}$ and $P 2_{1} / m$ were consistent with the pattern of systematic absences in the intensity data. $\mathrm{P} 2_{1}$ was confirmed by obtaining a physically sensible and stable solution and refinement of the structure. The structure solution was checked for missed symmetry elements with the ADDSYM program run from PLATON ${ }^{4-7}$, which found none. The absolute structure (Flack) parameter after the final refinement cycle was 0.04(11). The asymmetric unit consists of one molecule. Non-hydrogen atoms were refined with anisotropic displacement parameters. Hydrogen atoms were placed in geometrically idealized positions and included as riding atoms.

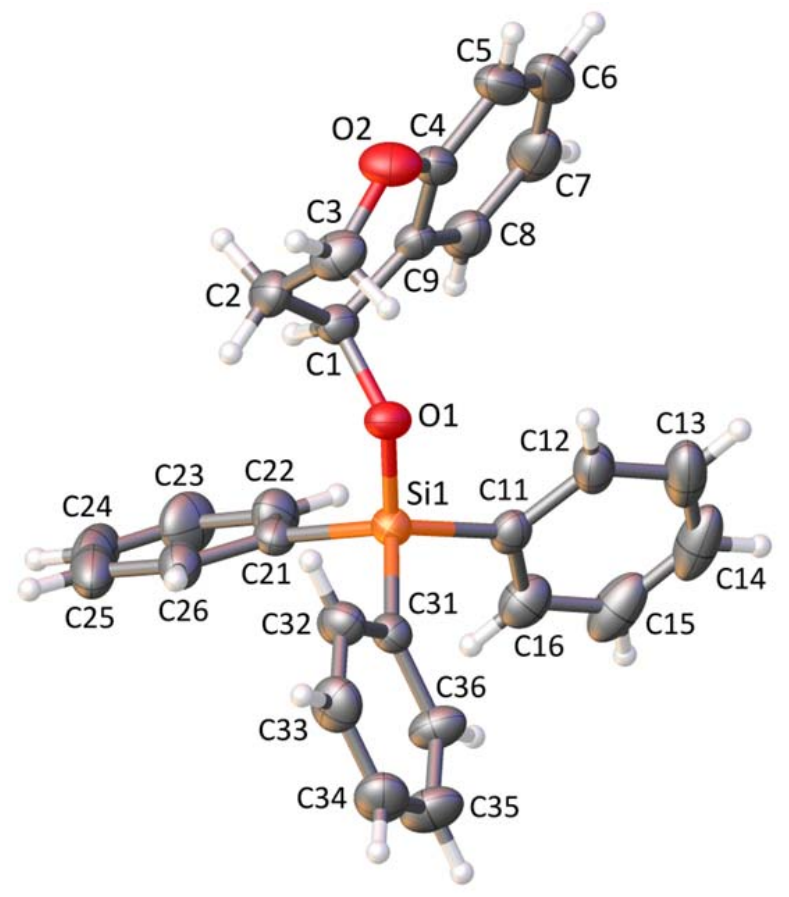

Figure S1. Molecular structure. Displacement ellipsoids drawn at the 30\% probability level. The crystal is enantiomerically pure; all molecules in the crystal are identical. C1 determined by the $\mathrm{X}$-ray data to have the " $\mathrm{S}$ " conformation. 
X-Ray Structure Determination of $(\boldsymbol{R})$-5. X-ray intensity data from a colorless plate crystal were collected at $200(2) \mathrm{K}$ using a diffractometer (Mo Ka radiation, $I=0.71073 \AA)^{1}$. The raw area detector data frames were reduced with the Bruker SAINT+ program ${ }^{1}$. Final unit cell parameters were determined by least-squares refinement of 4370 reflections from the data set. Direct methods structure solution, difference Fourier calculations and full-matrix least-squares refinement against $F^{2}$ were performed with SHELXS $/ \mathrm{L}^{2}$ as implemented in OLEX2. ${ }^{3}$

The compound crystallizes in the monoclinic system. Intensity statistics indicated an acentric structure (mean $\left|E^{*} E-1\right|=0.752$ ). The space group $P 2_{1}$ and $P 2_{1} / m$ were consistent with the pattern of systematic absences in the intensity data. $\mathrm{P} 2_{1}$ was confirmed by obtaining a physically sensible and stable solution and refinement of the structure. The structure solution was checked for missed symmetry elements with the ADDSYM program run from PLATON ${ }^{4-7}$, which found none. The absolute structure (Flack) parameter after the final refinement cycle was 0.03(9). The asymmetric unit consists of one molecule. Non-hydrogen atoms were refined with anisotropic displacement parameters. Hydrogen atoms were placed in geometrically idealized positions and included as riding atoms.

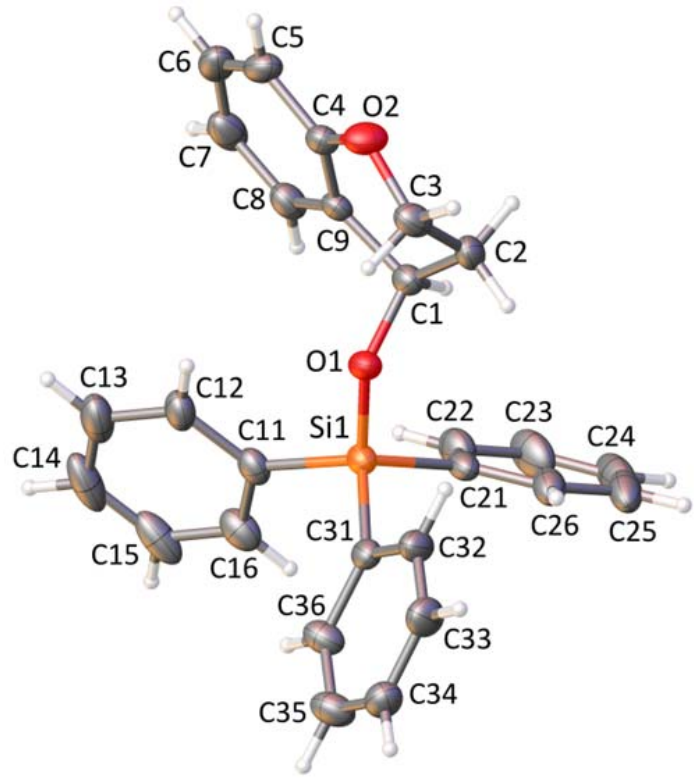

Figure S2. Molecular structure. Displacement ellipsoids drawn at the 30\% probability level. The crystal is enantiomerically pure; all molecules in the crystal are identical. C1 determined by the $\mathrm{X}$-ray data to have the " $\mathrm{R}$ " conformation 
X-Ray Structure Determination of 7. X-ray intensity data from a colorless plate crystal were measured at $100(2) \mathrm{K}$ using a diffractometer (Mo Ka radiation, $\mathrm{I}=0.71073 \AA)^{1}$. The raw area detector data frames were reduced and corrected for absorption effects with the SAINT+ and SADABS programs ${ }^{2}$. Final unit cell parameters were determined by least-squares refinement of 6635 reflections from the data set. Direct methods structure solution, difference Fourier calculations and full-matrix least-squares refinement against $F^{2}$ were performed with SHELXS/ ${ }^{2}$ as implemented in OLEX2. ${ }^{3}$

The compound crystallizes in the triclinic crystal system. The space group P1 (No. 1) was eventually confirmed by obtaining a stable and sensible solution and refinement of the structure. The absolute structure (Flack) parameter refined to 0.02(8), indicating the correct crystal handedness and the absence of inversion twinning. A solution in space group P-1 (No. 2) showed that adjacent tri(phenyl)silicon groupings are actually consistent with the inversion symmetry in $\mathrm{P}-1$, but that the $-\mathrm{C}_{10} \mathrm{H}_{17}$ substituents are not, as the inversion operation imposes two-fold disorder on this part of the molecule. The structure is therefore partly but not entirely consistent with P-1 and is pseudosymmetric. The asymmetric unit in the correct space group P1 (No. 1) consists of two independent, chemically identical but conformationally distinct molecules. Non-hydrogen atoms were refined with anisotropic displacement parameters. Hydrogen atoms were placed in geometrically idealized positions and included as riding atoms.

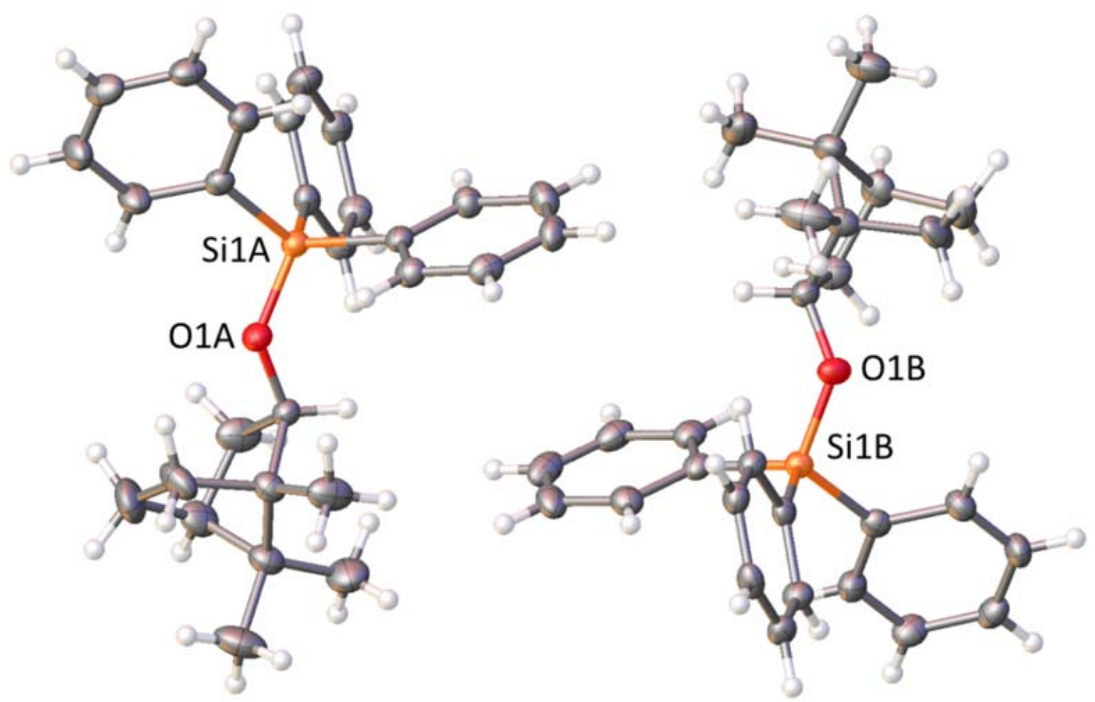

Figure S3. Asymmetric unit of the crystal. Two independent, chemically identical but conformationally distinct molecules, labeled " $\mathrm{A}$ " and "B". 


\begin{tabular}{|c|c|}
\hline Identification code & PJSI19 \\
\hline Empirical formula & $\mathrm{C}_{27} \mathrm{H}_{24} \mathrm{O}_{2} \mathrm{Si}$ \\
\hline Formula weight & 408.55 \\
\hline Temperature/K & $296(2)$ \\
\hline Crystal system & monoclinic \\
\hline Space group & $\mathrm{P} 21$ \\
\hline $\mathrm{a} / \AA ̊ \AA$ & $10.9603(11)$ \\
\hline $\mathrm{b} / \AA ̊ \AA$ & $7.6260(8)$ \\
\hline$c / \AA ̊$ & $13.2439(14)$ \\
\hline$\alpha /^{\circ}$ & 90.00 \\
\hline$\beta /^{\circ}$ & $92.830(3)$ \\
\hline$\gamma /{ }^{\circ}$ & 90.00 \\
\hline Volume/Å3 & $1105.6(2)$ \\
\hline Z & 2 \\
\hline pcalcmg/mm3 & 1.227 \\
\hline $\mathrm{m} / \mathrm{mm} 1$ & 0.127 \\
\hline$F(000)$ & 432.0 \\
\hline Crystal size/mm3 & $0.38 \times 0.34 \times 0.12$ \\
\hline $2 \Theta$ range for data collection & 3.08 to $50.02^{\circ}$ \\
\hline Index ranges & $-12 \leq h \leq 13,-9 \leq k \leq 9,-15 \leq 1 \leq 15$ \\
\hline Reflections collected & 12988 \\
\hline Independent reflections & $3883[R($ int $)=0.0597]$ \\
\hline Data/restraints/parameters & $3883 / 1 / 271$ \\
\hline Goodness-of-fit on F2 & 0.860 \\
\hline Final $R$ indexes $[I>=2 \sigma(I)]$ & $\mathrm{R} 1=0.0421, \mathrm{wR} 2=0.0658$ \\
\hline Final $R$ indexes [all data] & $R 1=0.0634, w R 2=0.0721$ \\
\hline Largest diff. peak/hole / e Å-3 & $0.24 /-0.16$ \\
\hline Flack parameter & $0.04(11)$ \\
\hline
\end{tabular}




\begin{tabular}{|c|c|}
\hline Identification code & mmm119am \\
\hline Empirical formula & $\mathrm{C}_{27} \mathrm{H}_{24} \mathrm{O}_{2} \mathrm{Si}$ \\
\hline Formula weight & 408.55 \\
\hline Temperature/K & $200(2)$ \\
\hline Crystal system & monoclinic \\
\hline Space group & $\mathrm{P} 21$ \\
\hline $\mathrm{a} / \AA ̊$ & $11.0421(6)$ \\
\hline $\mathrm{b} / \AA ̊ \AA$ & $7.5831(4)$ \\
\hline$c / \AA ̊$ & $12.9825(7)$ \\
\hline$\alpha /^{\circ}$ & 90.00 \\
\hline$\beta /^{\circ}$ & $92.9590(10)$ \\
\hline$\gamma /{ }^{\circ}$ & 90.00 \\
\hline Volume/Å3 & $1085.62(10)$ \\
\hline Z & 2 \\
\hline pcalcmg/mm3 & 1.250 \\
\hline $\mathrm{m} / \mathrm{mm} 1$ & 0.129 \\
\hline$F(000)$ & 432.0 \\
\hline Crystal size/mm3 & $0.32 \times 0.28 \times 0.12$ \\
\hline $2 \Theta$ range for data collection & 3.14 to $52.74^{\circ}$ \\
\hline Index ranges & $-13 \leq h \leq 13,-9 \leq k \leq 9,-16 \leq \mathrm{I} \leq 16$ \\
\hline Reflections collected & 15452 \\
\hline Independent reflections & $4452[\mathrm{R}$ (int) $=0.0422]$ \\
\hline Data/restraints/parameters & $4452 / 1 / 271$ \\
\hline Goodness-of-fit on F2 & 0.955 \\
\hline Final $R$ indexes $[I>=2 \sigma(I)]$ & $\mathrm{R} 1=0.0376, w R 2=0.0740$ \\
\hline Final $R$ indexes [all data] & $\mathrm{R} 1=0.0458, w R 2=0.0772$ \\
\hline Largest diff. peak/hole / e Å-3 & $0.20 /-0.17$ \\
\hline Flack parameter & $0.03(9)$ \\
\hline
\end{tabular}




\begin{tabular}{|c|c|}
\hline Identification code & CIS-497 \\
\hline Empirical formula & $\mathrm{C}_{28} \mathrm{H}_{32} \mathrm{OSi}$ \\
\hline Formula weight & 412.63 \\
\hline Temperature/K & $100(2)$ \\
\hline Crystal system & triclinic \\
\hline Space group & P1 (No. 1) \\
\hline $\mathrm{a} / \AA ̊$ & $10.4387(6)$ \\
\hline$b / \AA$ & $11.1375(6)$ \\
\hline$c / \AA$ & $11.3286(6)$ \\
\hline$\alpha /^{\circ}$ & $91.3171(12)$ \\
\hline$\beta /^{\circ}$ & $113.6586(10)$ \\
\hline$\gamma /{ }^{\circ}$ & $104.2860(11)$ \\
\hline Volume/Å3 & $1157.97(11)$ \\
\hline Z & 2 \\
\hline pcalcmg/mm3 & 1.183 \\
\hline $\mathrm{m} / \mathrm{mm} 1$ & 0.118 \\
\hline$F(000)$ & 444.0 \\
\hline Crystal size/mm3 & $0.54 \times 0.36 \times 0.06$ \\
\hline $2 \Theta$ range for data collection & 3.8 to $52.78^{\circ}$ \\
\hline Index ranges & $-13 \leq h \leq 13,-13 \leq k \leq 13,-14 \leq \mathrm{I} \leq 14$ \\
\hline Reflections collected & 17804 \\
\hline Independent reflections & $9341[R($ int $)=0.0259]$ \\
\hline Data/restraints/parameters & $9341 / 3 / 547$ \\
\hline Goodness-of-fit on F2 & 1.030 \\
\hline Final $R$ indexes $[I>=2 \sigma(I)]$ & $\mathrm{R} 1=0.0418, w R 2=0.0961$ \\
\hline Final $R$ indexes [all data] & $\mathrm{R} 1=0.0480, w R 2=0.0999$ \\
\hline Largest diff. peak/hole / e Å-3 & $0.45 /-0.18$ \\
\hline Flack parameter & $0.02(8)$ \\
\hline
\end{tabular}




\section{Molecular Modeling Information}

Conformer searches were carried out using the Monte Carlo for Complex Chemical System (MCCCS) Towhee ${ }^{8}$ plug-in built into Scienomics' Materials Processes and Simulations (MAPS) platform ${ }^{9}$. The conformers with energies within $2 \mathrm{kcal} / \mathrm{mol}$ relative to the lowest energy conformer were selected and the structures were optimized using DFT (B3LYP) with 6-311++** basis set in $\operatorname{Spartan}^{10}$. For all optimized structures, frequency calculations were carried out at the same level of theory to confirm that the conformers were stable. A Boltzmann distribution of the selected conformers was calculated on the basis of $\Delta \mathrm{G}$ and $\mathrm{T}=298 \mathrm{~K}$.

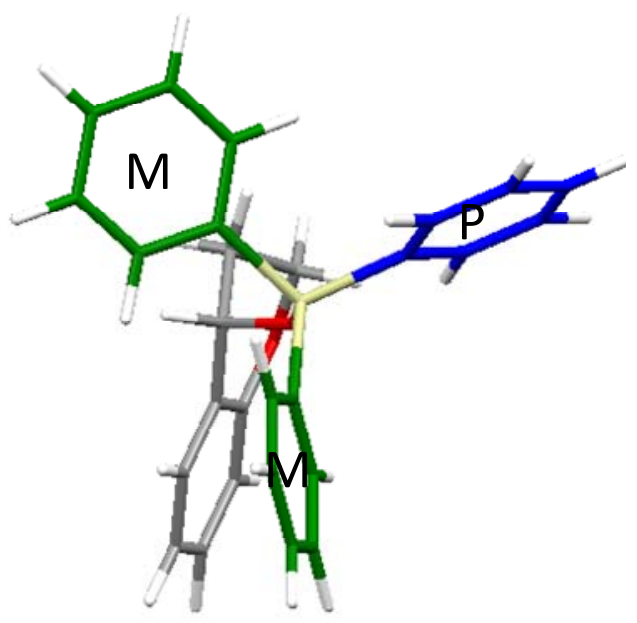

MPM table 4, entry 1

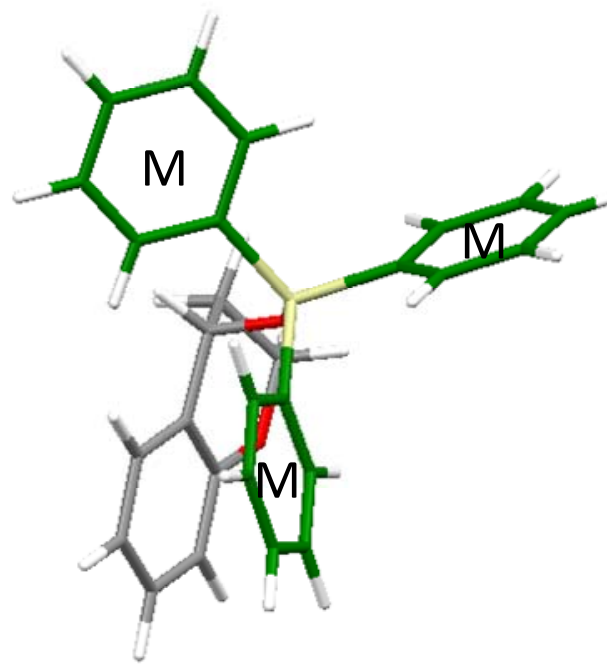

MMM table 4, entry 3

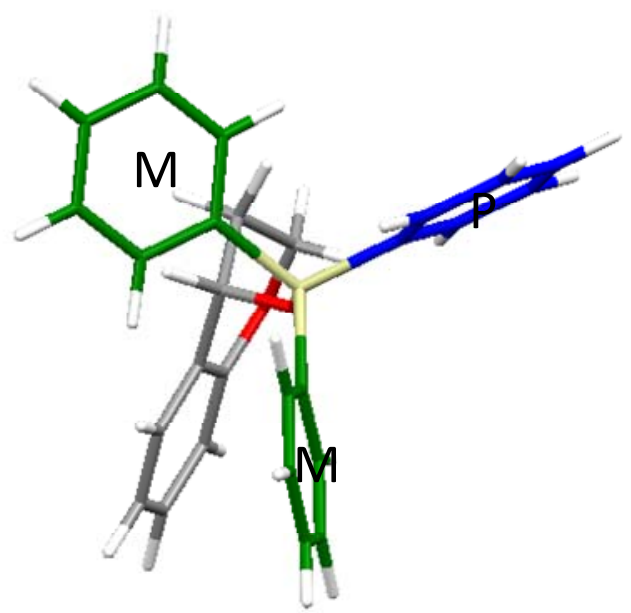

MPM table 4, entry 2

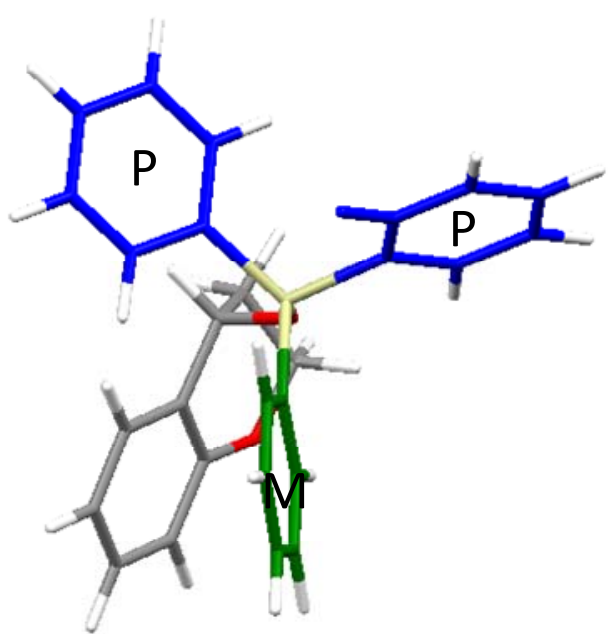

PMP table 4, entry 4

Figure S5. Calculated conformers of (S)-5 with the lowest energy 
Table S4. Absolute Energies of the conformers of (S)-5:

$\begin{array}{ll}\text { MPM } 1 & -1483.64749 \mathrm{au} \\ \text { MPM 2 } & -1450.64854 \mathrm{au} \\ \text { MMM } & -1450.64632 \mathrm{au} \\ \text { PMP } & -1450.64487 \mathrm{au}\end{array}$

Table S5. Cartesian Coordinates of the conformers of (S)-5:

$\begin{array}{cccc}\text { MPM } 1 & & & \\ & \mathrm{X} & \mathrm{Y} & \mathrm{Z} \\ \mathrm{Si} & -0.079778 & 0.399407 & -0.692982 \\ \mathrm{O} & 1.008222 & -0.509593 & 0.299019 \\ \mathrm{O} & 2.933222 & -2.649593 & 2.368019 \\ \mathrm{C} & 0.618222 & -1.777593 & 0.771019 \\ \mathrm{H} & -0.387778 & -2.114593 & 0.431019 \\ \mathrm{C} & 1.626222 & -2.812593 & 0.267019 \\ \mathrm{H} & 1.263222 & -3.838593 & 0.499019 \\ \mathrm{H} & 1.740222 & -2.733593 & -0.835982 \\ \mathrm{C} & 2.978222 & -2.596593 & 0.956019 \\ \mathrm{H} & 3.676223 & -3.385593 & 0.606019 \\ \mathrm{H} & 3.416222 & -1.621593 & 0.646019 \\ \mathrm{C} & 1.803222 & -2.123593 & 2.977019 \\ \mathrm{C} & 1.814222 & -2.034593 & 4.376019 \\ \mathrm{H} & 2.694222 & -2.336593 & 4.930019 \\ \mathrm{C} & 0.701222 & -1.558593 & 5.072019 \\ \mathrm{H} & 0.725222 & -1.494593 & 6.152019 \\ \mathrm{C} & -0.444778 & -1.170593 & 4.375019 \\ \mathrm{H} & -1.307778 & -0.803593 & 4.915019 \\ \mathrm{C} & -0.477778 & -1.254593 & 2.981019 \\ \mathrm{H} & -1.371778 & -0.943593 & 2.456019 \\ \mathrm{C} & 0.636222 & -1.735593 & 2.274019 \\ \mathrm{C} & -0.977778 & 1.637408 & 0.356019 \\ \mathrm{C} & -2.100778 & 2.311408 & -0.150981 \\ \mathrm{H} & -2.476778 & 2.102408 & -1.144982 \\ \mathrm{C} & -2.758778 & 3.268408 & 0.626019 \\ \mathrm{H} & -3.625778 & 3.781408 & 0.231019 \\ \mathrm{C} & -2.296778 & 3.565408 & 1.910019 \\ \mathrm{H} & -2.806778 & 4.307408 & 2.511019 \\ \mathrm{C} & -1.169778 & 2.910408 & 2.415019 \\ \mathrm{H} & -0.807778 & 3.146408 & 3.408019 \\ \mathrm{C} & -0.505778 & 1.956408 & 1.638019 \\ \mathrm{H} & 0.372222 & 1.474408 & 2.048019\end{array}$




$\begin{array}{llll}\text { C } & -1.242778 & -0.640593 & -1.702982 \\ \text { C } & -0.839778 & -1.151593 & -2.945982 \\ \text { H } & 0.148222 & -0.946593 & -3.341982 \\ \text { C } & -1.718778 & -1.928593 & -3.706982 \\ \text { H } & -1.408778 & -2.308593 & -4.671982 \\ \text { C } & -2.998778 & -2.215593 & -3.222982 \\ \text { H } & -3.679778 & -2.813593 & -3.813982 \\ \text { C } & -3.395778 & -1.733593 & -1.972982 \\ \text { H } & -4.383778 & -1.961593 & -1.594982 \\ \text { C } & -2.519778 & -0.955593 & -1.210982 \\ \text { H } & -2.848778 & -0.600593 & -0.242981 \\ \text { C } & 0.956222 & 1.429407 & -1.836982 \\ \text { C } & 2.352222 & 1.470408 & -1.695982 \\ \text { H } & 2.857222 & 0.897407 & -0.927982 \\ \text { C } & 3.124222 & 2.257408 & -2.555982 \\ \text { H } & 4.200223 & 2.283408 & -2.444982 \\ \text { C } & 2.508222 & 3.012408 & -3.557982 \\ \text { H } & 3.107222 & 3.621408 & -4.221982 \\ \text { C } & 1.118222 & 2.979408 & -3.700982 \\ \text { H } & 0.641222 & 3.565408 & -4.475982 \\ \text { C } & 0.344222 & 2.192408 & -2.843982 \\ \text { H } & -0.730778 & 2.182408 & -2.977982\end{array}$

$\begin{array}{cccc}\text { MPM 2 } & \text { X } & \text { Y } & \text { Z } \\ \text { Si } & -0.585148 & 0.513537 & 0.333389 \\ \text { O } & 0.104852 & -0.363463 & -0.988611 \\ \text { O } & 1.134852 & -2.716463 & -3.494611 \\ \text { C } & 0.482852 & -1.706463 & -0.807611 \\ \text { H } & 0.462852 & -2.061463 & 0.247389 \\ \text { C } & -0.462148 & -2.603463 & -1.610611 \\ \text { H } & -0.295148 & -3.668463 & -1.336611 \\ \text { H } & -1.520148 & -2.353463 & -1.377611 \\ \text { C } & -0.194148 & -2.428463 & -3.108611 \\ \text { H } & -0.870148 & -3.110463 & -3.665611 \\ \text { H } & -0.449148 & -1.393463 & -3.428611 \\ \text { C } & 2.132852 & -2.283463 & -2.634611 \\ \text { C } & 3.453852 & -2.352463 & -3.096611 \\ \text { H } & 3.658852 & -2.709463 & -4.098611 \\ \text { C } & 4.518852 & -1.955463 & -2.283611 \\ \text { H } & 5.532852 & -2.007463 & -2.656611 \\ \text { C } & 4.274852 & -1.489463 & -0.989611 \\ \text { H } & 5.099852 & -1.178463 & -0.362611 \\ \text { C } & 2.966852 & -1.423463 & -0.507611 \\ \text { H } & 2.792852 & -1.050463 & 0.495389 \\ \text { C } & 1.892852 & -1.831463 & -1.314611 \\ \text { C } & 0.668852 & 1.779537 & 0.847389 \\ \text { C } & 0.534852 & 2.458537 & 2.068389\end{array}$




\begin{tabular}{|c|c|c|c|}
\hline & -0.292148 & 250537 & \\
\hline & 1.481852 & 3.412537 & 2.452389 \\
\hline & 1.379852 & 3.925537 & 400389 \\
\hline & 2.560852 & 3.705537 & 1.614389 \\
\hline & 3.294852 & .443537 & 912389 \\
\hline & 2.690852 & 3.046537 & 0.389389 \\
\hline & 3.523852 & 3.277537 & -0.262611 \\
\hline & 1.746852 & 2.090537 & 0.003389 \\
\hline & 1.871852 & 1.598537 & -0.953611 \\
\hline & -1.114148 & -0.535463 & 1.774389 \\
\hline & -2.398148 & -1.100463 & 1.80938 \\
\hline & -3.112148 & -0.915463 & 1.016389 \\
\hline & -2.778148 & -1.918463 & $2.87838 \mathrm{~s}$ \\
\hline & -3.771148 & -2.347463 & 2.90538 \\
\hline & -1.875148 & -2.188463 & 3.910389 \\
\hline & -2.170148 & -2.822463 & 4.73638 \\
\hline & -0.588148 & -1.647463 & 3.87038 \\
\hline & 0.115852 & -1.865463 & 4.66338 \\
\hline & -0.205148 & -0.830463 & 2.80238 \\
\hline & 0.804852 & -0.438463 & 2.78738 \\
\hline & -2.037148 & 1.437537 & -0.359611 \\
\hline & -2.379148 & 1.311537 & -1.71661 \\
\hline & -1.811148 & 0.677537 & -2.38561 \\
\hline & -3.472148 & 2.010537 & -2.23561 \\
\hline & -3.730148 & 1.910537 & -3.28261 \\
\hline & -4.232148 & 2.839537 & -1.40561 \\
\hline & -5.078148 & 3.379537 & -1.81061 \\
\hline & -3.899148 & 2.967537 & -0.05461 \\
\hline & -4.489148 & 3.608537 & 0.58738 \\
\hline & -2.806148 & 2.269537 & 0.46838 \\
\hline & -2.570148 & 2.382537 & 1.519 \\
\hline
\end{tabular}

$\begin{array}{cccc}\text { MMM } & \text { X } & \text { Y } & \text { Z } \\ \text { Si } & 0.422889 & -0.283944 & 0.686630 \\ \text { O } & 1.014889 & 0.592056 & -0.683370 \\ \text { O } & 0.936889 & 1.784056 & -3.930371 \\ \text { C } & 0.474889 & 1.846056 & -1.024370 \\ \text { H } & 0.138889 & 2.428056 & -0.136370 \\ \text { C } & 1.597889 & 2.648056 & -1.687370 \\ \text { H } & 1.258889 & 3.688056 & -1.888371 \\ \text { H } & 2.481889 & 2.703056 & -1.014370 \\ \text { C } & 1.994889 & 1.980056 & -3.013371 \\ \text { H } & 2.760889 & 2.615056 & -3.505371 \\ \text { H } & 2.478889 & 0.999056 & -2.816371 \\ \text { C } & -0.330111 & 1.574056 & -3.404371 \\ \text { C } & -1.362111 & 1.321056 & -4.318371\end{array}$




$\begin{array}{llll}\text { H } & -1.143111 & 1.248056 & -5.376371 \\ \mathrm{C} & -2.681111 & 1.168056 & -3.883371 \\ \mathrm{H} & -3.468111 & 0.971056 & -4.599371 \\ \mathrm{C} & -2.986111 & 1.284056 & -2.527371 \\ \mathrm{H} & -4.008111 & 1.173056 & -2.190371 \\ \mathrm{C} & -1.971111 & 1.535056 & -1.603371 \\ \mathrm{H} & -2.230111 & 1.611056 & -0.557370 \\ \mathrm{C} & -0.638111 & 1.668056 & -2.025371 \\ \mathrm{C} & -1.107111 & -1.207945 & 0.190630 \\ \mathrm{C} & -1.274111 & -1.618945 & -1.141370 \\ \mathrm{H} & -0.530111 & -1.388945 & -1.895370 \\ \mathrm{C} & -2.422111 & -2.317945 & -1.525370 \\ \mathrm{H} & -2.555111 & -2.612945 & -2.558371 \\ \mathrm{C} & -3.397111 & -2.636945 & -0.576370 \\ \mathrm{H} & -4.284111 & -3.180945 & -0.874370 \\ \mathrm{C} & -3.221111 & -2.262945 & 0.757630 \\ \mathrm{H} & -3.969111 & -2.522945 & 1.495630 \\ \mathrm{C} & -2.078111 & -1.555945 & 1.142630 \\ \mathrm{H} & -1.961111 & -1.287945 & 2.185630 \\ \mathrm{C} & 0.199889 & 0.719056 & 2.238630 \\ \mathrm{C} & 1.187889 & 0.729056 & 3.236630 \\ \mathrm{H} & 2.102889 & 0.161056 & 3.127630 \\ \mathrm{C} & 0.999889 & 1.466056 & 4.410630 \\ \mathrm{H} & 1.758889 & 1.458056 & 5.182630 \\ \mathrm{C} & -0.169111 & 2.210056 & 4.592630 \\ \mathrm{H} & -0.316111 & 2.777056 & 5.502630 \\ \mathrm{C} & -1.150111 & 2.218056 & 3.598630 \\ \mathrm{H} & -2.057111 & 2.793056 & 3.738630 \\ \mathrm{C} & -0.966111 & 1.477056 & 2.427630 \\ \mathrm{H} & -1.751111 & 1.490056 & 1.685630 \\ \mathrm{C} & 1.745889 & -1.563945 & 0.927630 \\ \mathrm{C} & 3.063889 & -1.290945 & 0.522630 \\ \mathrm{H} & 3.329889 & -0.343944 & 0.069630 \\ \mathrm{C} & 4.067889 & -2.248945 & 0.690630 \\ \mathrm{H} & 5.077889 & -2.035945 & 0.364630 \\ \mathrm{C} & 3.767889 & -3.479945 & 1.278630 \\ \mathrm{H} & 4.544889 & -4.222945 & 1.403630 \\ \mathrm{H} & 2.464889 & -3.749945 & 1.706630 \\ & 2.235889 & -4.701945 & 2.168630 \\ & 0.458889 & -3.025945 & 1.883630\end{array}$

$\begin{array}{lccc}\text { PMP } 1 & & & \\ & \text { X } & Y & \text { Z } \\ \text { Si } & -0.484870 & -0.276019 & -0.582482 \\ \text { O } & -1.060870 & 0.601982 & 0.797519 \\ \text { O } & -0.637870 & 1.943982 & 4.058519 \\ \text { C } & -0.478870 & 1.845982 & 1.114519\end{array}$




\begin{tabular}{|c|c|c|c|}
\hline H & -0.209870 & 2.437982 & 0.210519 \\
\hline & -1.533870 & 2.666982 & 1.860519 \\
\hline & -1.179870 & 3.714982 & 1.987519 \\
\hline & -2.481871 & 2.698982 & 1.279519 \\
\hline & -1.785871 & 2.052982 & 3.243519 \\
\hline & -2.523871 & 2.689982 & 3.775519 \\
\hline & -2.249871 & 1.047982 & 3.139519 \\
\hline 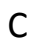 & 0.560130 & 1.661982 & 3.418519 \\
\hline 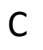 & 1.677130 & 1.438982 & 4.234519 \\
\hline & 1.573130 & 1.449982 & 5.311519 \\
\hline 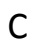 & 2.936130 & 1.204982 & 3.675519 \\
\hline 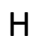 & 3.790130 & 1.030982 & 4.317519 \\
\hline f & 3.095130 & 1.208982 & 2.289519 \\
\hline 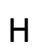 & 4.071130 & 1.032982 & 1.855519 \\
\hline 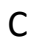 & 1.995130 & 1.439982 & 1.461519 \\
\hline 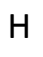 & 2.142130 & 1.441982 & 0.390519 \\
\hline & 0.722130 & 1.658982 & 2.011519 \\
\hline 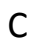 & 0.800130 & -1.510019 & -0.065482 \\
\hline - & 1.514130 & -2.232019 & -1.034482 \\
\hline$y$ & 1.336130 & -2.076019 & -2.091482 \\
\hline - & 2.480130 & -3.166019 & -0.650482 \\
\hline H & 3.034130 & -3.712019 & -1.402482 \\
\hline 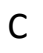 & 2.732130 & -3.394019 & 0.705519 \\
\hline 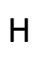 & 3.483130 & -4.115019 & 1.001519 \\
\hline C & 2.007130 & -2.699019 & 1.676519 \\
\hline-1 & 2.196130 & -2.882019 & 2.726519 \\
\hline 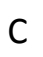 & 1.035130 & -1.769019 & 1.293519 \\
\hline 1 & 0.480130 & -1.255019 & 2.067519 \\
\hline C & 0.022130 & 0.813982 & -2.001482 \\
\hline F & -0.957870 & 1.524982 & -2.711482 \\
\hline $\mathrm{H}$ & -2.005871 & 1.447982 & -2.446482 \\
\hline 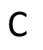 & -0.595870 & 2.340982 & -3.788482 \\
\hline H & -1.355870 & 2.878982 & -4.339482 \\
\hline C & 0.747130 & 2.460982 & -4.156482 \\
\hline 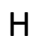 & 1.027130 & 3.089982 & -4.991482 \\
\hline C & 1.729130 & 1.768982 & -3.443482 \\
\hline 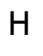 & 2.770130 & 1.863982 & -3.725482 \\
\hline 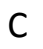 & 1.370130 & 0.951982 & -2.367482 \\
\hline $\mathrm{H}$ & 2.153130 & 0.429982 & -1.835482 \\
\hline C & -1.880870 & -1.349019 & -1.164482 \\
\hline 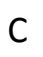 & -1.836871 & -1.935019 & -2.439482 \\
\hline 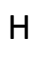 & -1.008870 & -1.750019 & -3.111482 \\
\hline 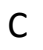 & -2.869871 & -2.776019 & -2.865482 \\
\hline 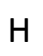 & -2.830871 & -3.224019 & -3.849482 \\
\hline C & -3.949871 & -3.042019 & -2.019482 \\
\hline $\mathrm{H}$ & -4.747871 & -3.695019 & -2.349482 \\
\hline 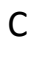 & -3.995871 & -2.469019 & -0.745482 \\
\hline $\mathrm{H}$ & -4.830871 & -2.680019 & -0.089481 \\
\hline
\end{tabular}




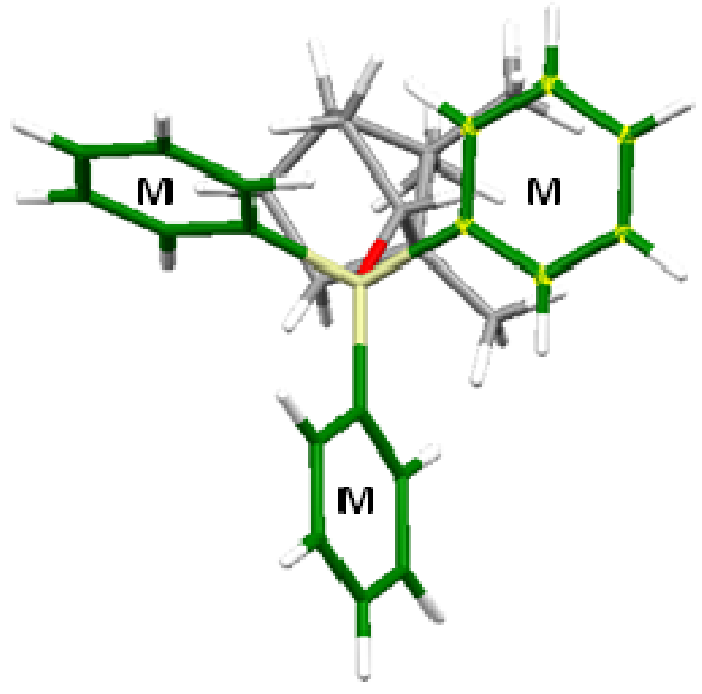

MMM table 4, entry 5

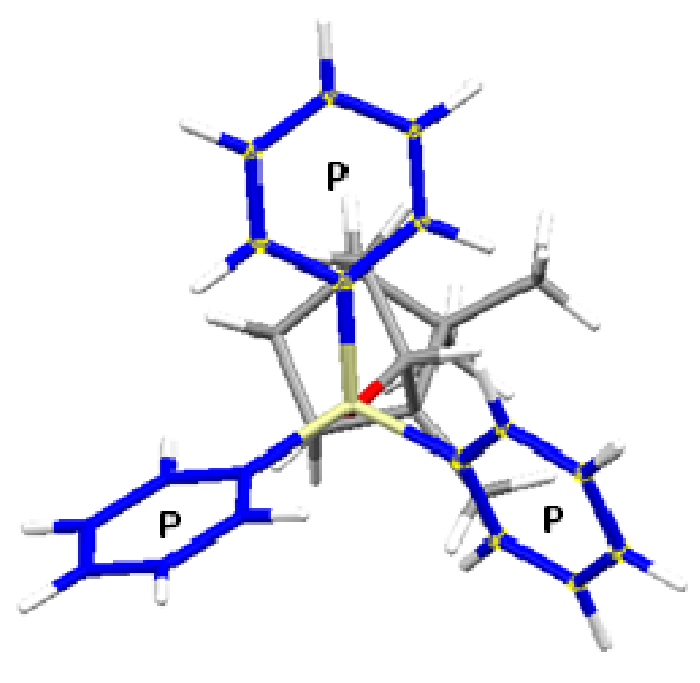

PMP table 4, entry 6

Figure S4. Calculated conformers of $\mathbf{7}$ in a MMM and PPP helicity

Table S6. Absolute Energies of the conformers of 7

$\begin{array}{ll}\text { MMM } & -1450.68644 \mathrm{au} \\ \text { PPP } & -1450.68476 \mathrm{au}\end{array}$

Table S7. Cartesian Coordinates of the conformers of 7.

$\begin{array}{lccc}\text { MMM } & & & \\ & \mathrm{X} & \mathrm{Y} & \mathrm{Z} \\ \mathrm{Si} & 0.344742 & 0.376113 & -1.313565 \\ \mathrm{O} & -0.456258 & 0.375113 & 0.112436 \\ \mathrm{C} & -0.205258 & -0.328887 & 1.325436 \\ \mathrm{H} & 0.776742 & -0.321887 & 1.518436 \\ \mathrm{C} & -0.963258 & 0.314113 & 2.500436 \\ \mathrm{C} & -2.442258 & 0.389113 & 2.088436 \\ \mathrm{H} & -2.536258 & 0.759113 & 1.173436 \\ \text { H } & -2.957258 & 0.955113 & 2.717436\end{array}$




\begin{tabular}{|c|c|c|c|}
\hline & & -1.0 & \\
\hline & -3.645258 & -1.195887 & \\
\hline & 258 & -1.380887 & \\
\hline & .664258 & -1.838887 & 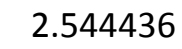 \\
\hline & 1.827258 & -2.753887 & 91 \\
\hline & -0.723258 & -1.796887 & 1.326 \\
\hline & -1.212258 & -2.002887 & 0.490 \\
\hline & 0.024742 & -2.438887 & 420 \\
\hline & -0.975258 & -0.856887 & 3.52843 \\
\hline & -0.369258 & 1.635113 & 2.947 \\
\hline & 0.572742 & 1.506113 & 318 \\
\hline & -0.859258 & 1.964113 & 3.73043 \\
\hline & -0.438258 & 2.287113 & 2.22043 \\
\hline & 0.40 & -1.323887 & 3.99143 \\
\hline & 0.935742 & -1.586887 & 3.21 \\
\hline & 0.301 & -2.091887 & 4.59243 \\
\hline & 0.85 & -0.593887 & 4.46543 \\
\hline & -1.801258 & -0.574887 & 4.787 \\
\hline & -1.3 & 0.105113 & 5.32943 \\
\hline & -1.8 & -1.399887 & 5.30843 \\
\hline & -2.68 & -0.249887 & 4.5 \\
\hline & -0.7 & 0887 & 2.6 \\
\hline & -2.1 & -0.291887 & -2.5 \\
\hline & -2.53 & 0.169113 & -1.79656 \\
\hline & -2.964 & -0.840887 & -3.50856 \\
\hline & $-3.9 c$ & -0.743887 & -3.44 \\
\hline & $-2.42 C$ & -1.527887 & -4.57356 \\
\hline & -2.98 & -1.906887 & -5.23556 \\
\hline & -1.05 & -1.664887 & -4.6755 \\
\hline & -0.67 & -2.140887 & -5.40256 \\
\hline & -0.230 & -1.096887 & -3.69 \\
\hline & 0.7 & -1.1 & -3.7 \\
\hline & 0.74 & 2.155113 & -1.71 \\
\hline & 1.15 & 3.023113 & -0.69 \\
\hline & & 2.71 & 0.20 \\
\hline & 1.520 & 4.324113 & -0.969 \\
\hline & 1.78 & 4.901113 & -0.261 \\
\hline & 1.508742 & 4.788113 & -2.271 \\
\hline & 1.768 & 5.683113 & -2.457 \\
\hline & 1.117 & 3.955113 & -3.306 \\
\hline & 1.103742 & 4.275113 & -4.201 \\
\hline & 0.744742 & 2.642113 & -3.0235 \\
\hline & 0.486742 & 2.068113 & -3.73456 \\
\hline & 1.952742 & -0.585887 & -1.24856 \\
\hline & 1.961742 & -1.951887 & -0.95756 \\
\hline & 1.139742 & -2.397887 & -0.78956 \\
\hline & 3.155 & -2.674887 & -0.90956 \\
\hline & 3142742 & -3.603887 & 71056 \\
\hline
\end{tabular}




$\begin{array}{llll}\mathrm{C} & 4.355742 & -2.035887 & -1.152565 \\ \mathrm{H} & 5.169742 & -2.523887 & -1.113565 \\ \mathrm{C} & 4.373742 & -0.689887 & -1.449565 \\ \mathrm{H} & 5.199742 & -0.251887 & -1.613565 \\ \mathrm{C} & 3.183742 & 0.028113 & -1.508565 \\ \mathrm{H} & 3.206742 & 0.953113 & -1.729565\end{array}$

\begin{tabular}{cccc} 
PPP & & & \\
& $\mathrm{X}$ & $\mathrm{Y}$ & $\mathrm{Z}$ \\
$\mathrm{Si}$ & -0.294935 & -0.143258 & 1.341581 \\
$\mathrm{O}$ & 0.558065 & 0.131742 & -0.022419 \\
$\mathrm{C}$ & 0.278065 & -0.195258 & -1.390419 \\
$\mathrm{H}$ & -0.702936 & -0.346258 & -1.511419 \\
$\mathrm{C}$ & 0.763065 & 0.916742 & -2.351420 \\
$\mathrm{C}$ & 2.206065 & 1.249742 & -1.955420 \\
$\mathrm{H}$ & 2.281065 & 1.407742 & -0.981419 \\
$\mathrm{H}$ & 2.533065 & 2.049742 & -2.439420 \\
$\mathrm{C}$ & 2.997065 & -0.017258 & -2.377420 \\
$\mathrm{H}$ & 3.707065 & 0.205742 & -3.030420 \\
$\mathrm{H}$ & 3.407065 & -0.459258 & -1.591419 \\
$\mathrm{C}$ & 1.924065 & -0.905258 & -3.011420 \\
$\mathrm{H}$ & 2.277065 & -1.605258 & -3.630420 \\
$\mathrm{C}$ & 1.067065 & -1.447258 & -1.854419 \\
$\mathrm{H}$ & 1.635065 & -1.804258 & -1.126419 \\
$\mathrm{H}$ & 0.456065 & -2.161258 & -2.167420 \\
$\mathrm{C}$ & 0.974065 & 0.109742 & -3.666420 \\
$\mathrm{C}$ & -0.164936 & 2.115742 & -2.405420 \\
$\mathrm{H}$ & -1.050936 & 1.829742 & -2.710420 \\
$\mathrm{H}$ & 0.196065 & 2.779742 & -3.030420 \\
$\mathrm{H}$ & -0.237936 & 2.513742 & -1.513419 \\
$\mathrm{C}$ & -0.292936 & -0.516258 & -4.251420 \\
$\mathrm{H}$ & -0.779936 & -0.989258 & -3.544420 \\
$\mathrm{H}$ & -0.048936 & -1.148258 & -4.959420 \\
$\mathrm{H}$ & -0.862936 & 0.187742 & -4.625420 \\
$\mathrm{C}$ & 1.624065 & 0.915742 & -4.794420 \\
$\mathrm{H}$ & 1.024065 & 1.642742 & -5.063420 \\
$\mathrm{H}$ & 1.790065 & 0.328742 & -5.561420 \\
$\mathrm{H}$ & 2.472065 & 1.290742 & -4.479420 \\
$\mathrm{C}$ & 0.775065 & 0.591742 & 2.673581 \\
$\mathrm{C}$ & 2.171065 & 0.496742 & 2.615581 \\
$\mathrm{H}$ & 2.576065 & 0.050742 & 1.881581 \\
$\mathrm{C}$ & 2.977065 & 1.039742 & 3.610581 \\
$\mathrm{H}$ & 3.922065 & 0.958742 & 3.555581 \\
$\mathrm{C}$ & 2.398065 & 1.695742 & 4.677581 \\
$\mathrm{H}$ & 2.948065 & 2.077742 & 5.350581 \\
& 1.030065 & 1.795742 & 4.769581 \\
& 0.635065 & 2.236742 & 5.513581 \\
\hline & & & \\
$\mathrm{H}$ & &
\end{tabular}




$\begin{array}{llll}\text { H } & -0.711936 & 1.338742 & 3.853581 \\ \mathrm{C} & -1.953936 & 0.730742 & 1.300581 \\ \mathrm{C} & -2.015936 & 2.099742 & 0.977581 \\ \mathrm{H} & -1.212936 & 2.564742 & 0.773581 \\ \mathrm{C} & -3.216936 & 2.786742 & 0.950581 \\ \mathrm{H} & -3.235936 & 3.711742 & 0.739581 \\ \mathrm{C} & -4.390936 & 2.111742 & 1.237581 \\ \mathrm{H} & -5.220936 & 2.573742 & 1.208581 \\ \mathrm{C} & -4.366936 & 0.764742 & 1.566581 \\ \mathrm{H} & -5.173936 & 0.306742 & 1.771581 \\ \mathrm{C} & -3.149936 & 0.087742 & 1.596581 \\ \mathrm{H} & -3.135936 & -0.835258 & 1.823581 \\ \mathrm{C} & -0.636936 & -1.947258 & 1.693581 \\ \mathrm{C} & -1.075936 & -2.822258 & 0.695581 \\ \mathrm{H} & -1.138936 & -2.512258 & -0.200419 \\ \mathrm{C} & -1.422936 & -4.141258 & 0.985581 \\ \mathrm{H} & -1.707936 & -4.722258 & 0.291581 \\ \mathrm{C} & -1.353936 & -4.603258 & 2.298581 \\ \mathrm{H} & -1.593936 & -5.499258 & 2.501581 \\ \mathrm{C} & -0.934936 & -3.752258 & 3.301581 \\ \mathrm{H} & -0.892936 & -4.062258 & 4.197581 \\ \mathrm{C} & -0.574936 & -2.443258 & 3.005581 \\ \mathrm{H} & -0.278935 & -1.874258 & 3.705581\end{array}$




\section{Reference}

(1) In SMART Version 5.630, SAINT+ Version 6.45; Bruker Analytical X-ray Systems, Inc.: Madison, Wisconsin, USA, 2003.

(2) Sheldrick, G. M. Acta. Crystallogr. A. 2008, 64, 112.

(3) Dolomanov, O. V.; Bourhis, L. J.; Gildea, R. J.; Howard, J. A. K.; Puschmann, H. J. Appl. Crystallogr. 2009, 42, 339.

(4) Spek, A. L. PLATON, A Multipurpose Crystallographic Tool, Utrecht University, Utrecht, The Netherlands, 1998.

(5) Vandersluis, P.; Spek, A. L. Acta. Crystallogr. A. 1990, 46, 194.

(6) Spek, A. L. J. Appl. Crystallogr. 1988, 21, 578.

(7) Lepage, Y. J. Appl. Crystallogr. 1987, 20, 264.

(8) Martin, M. G. Mol. Simulat. 2013, 39, 1212.

(9) In Materials and Processes Simulations (MAPS); Scienomics SARL: Paris, France, 2004-2013.

(10) In Spartan 10, Program for Calculation of Molecular Properties; Wavefunction Inc.: Irvine, CA, USA, 2015. 


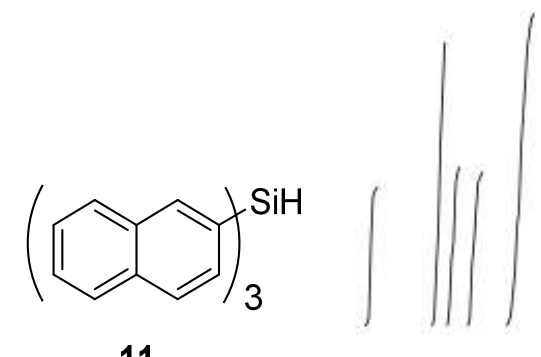

11

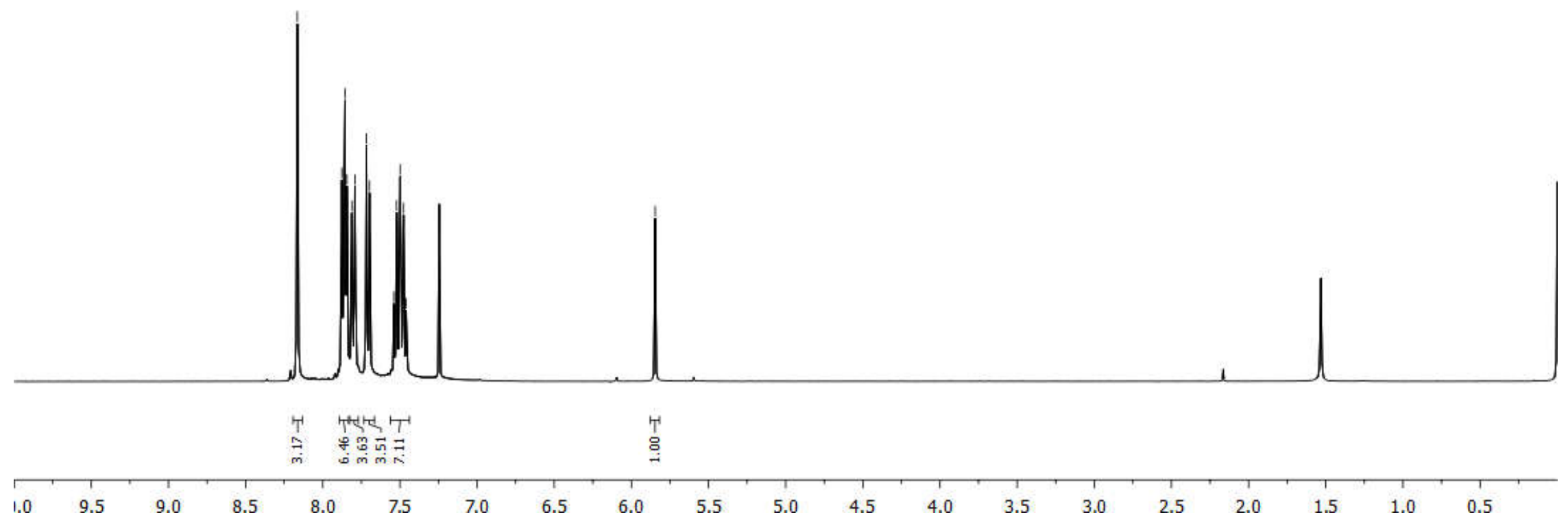




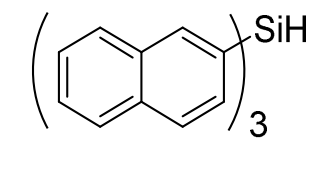

11

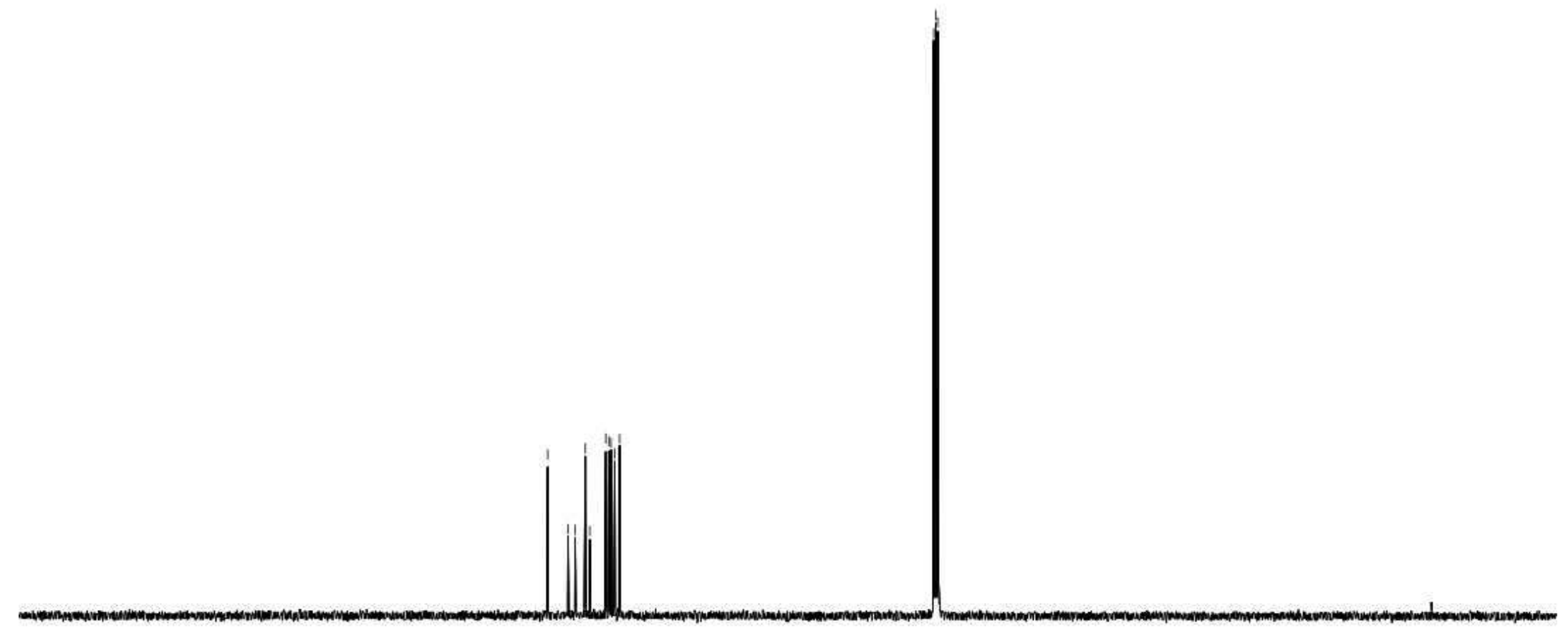




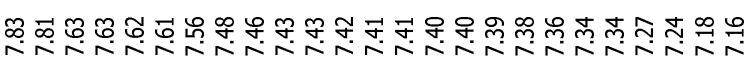

(

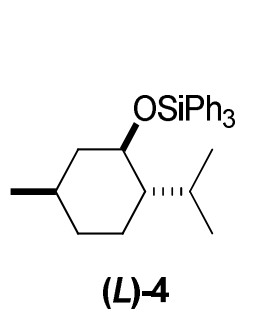

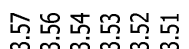

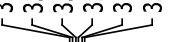

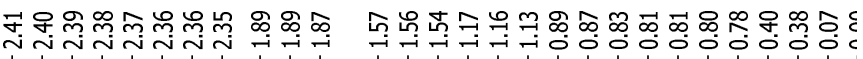
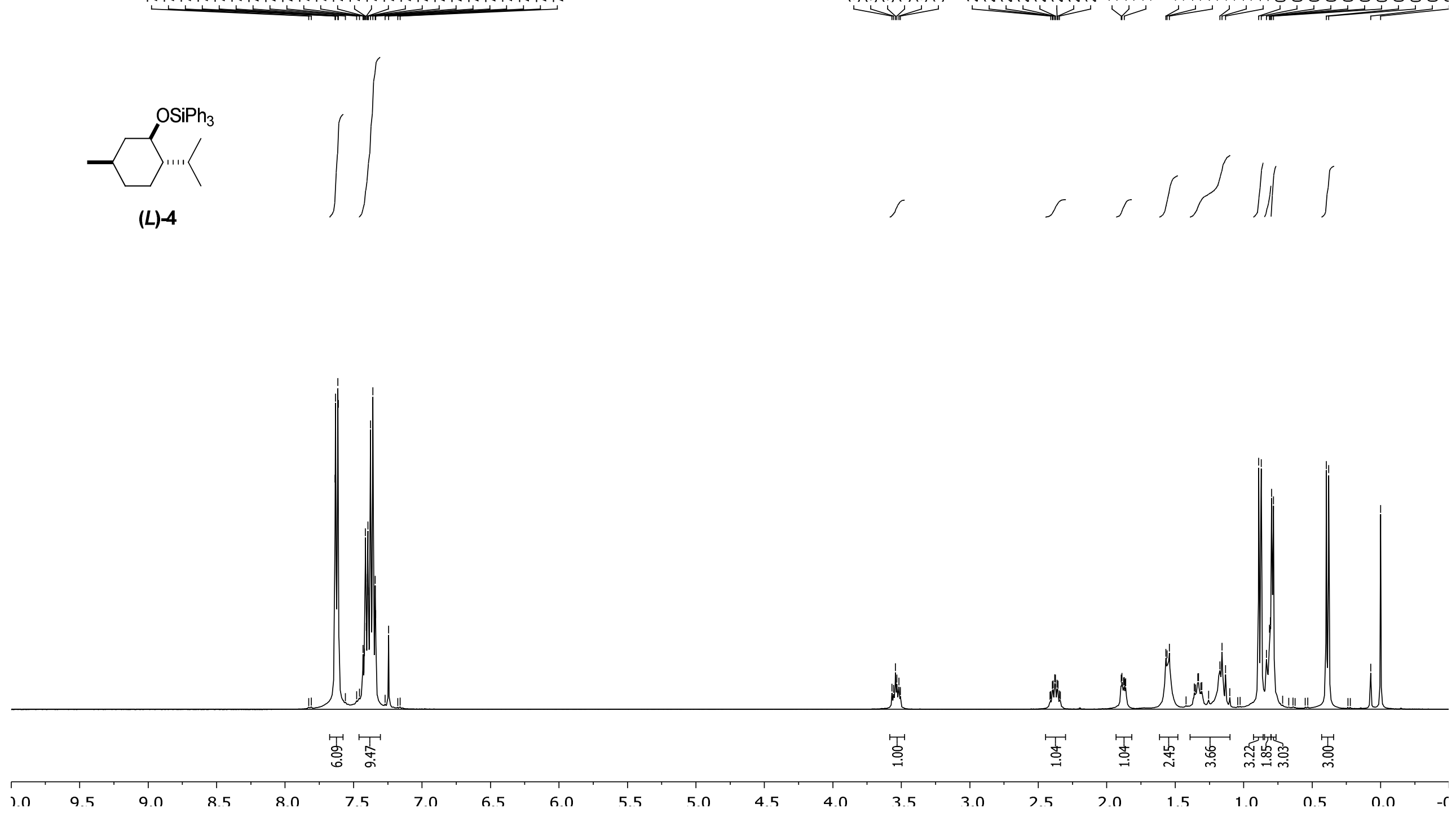


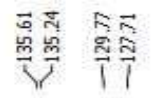

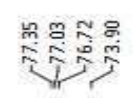

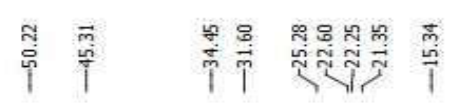
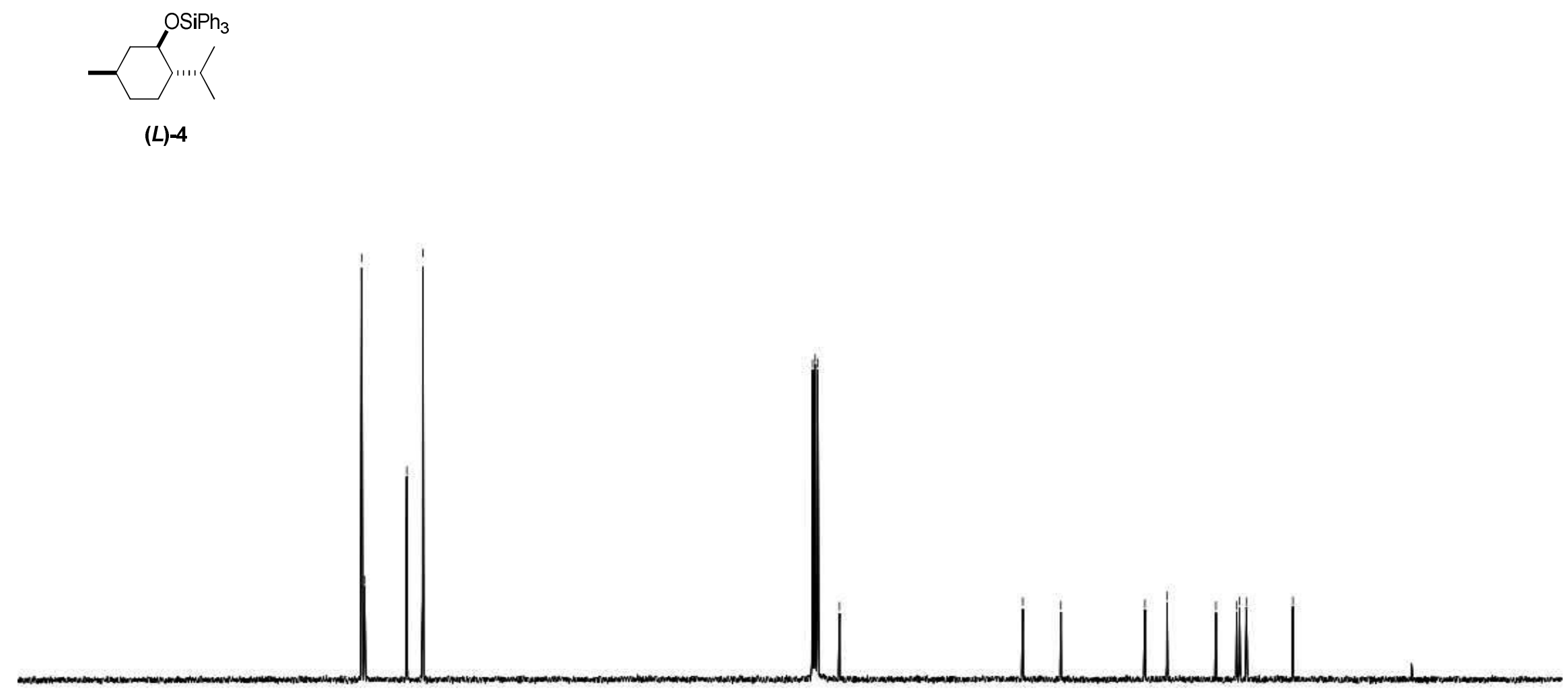


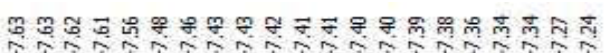

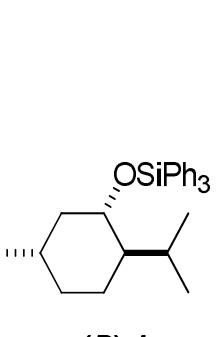

(D)-4

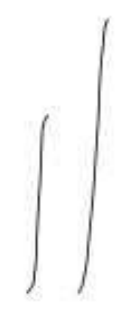

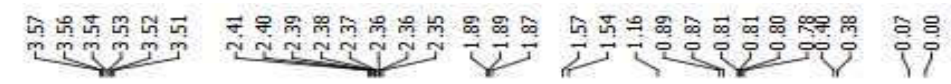

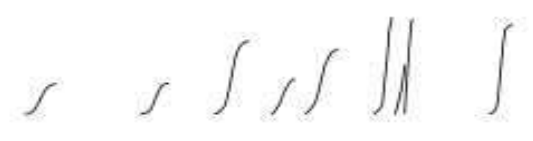

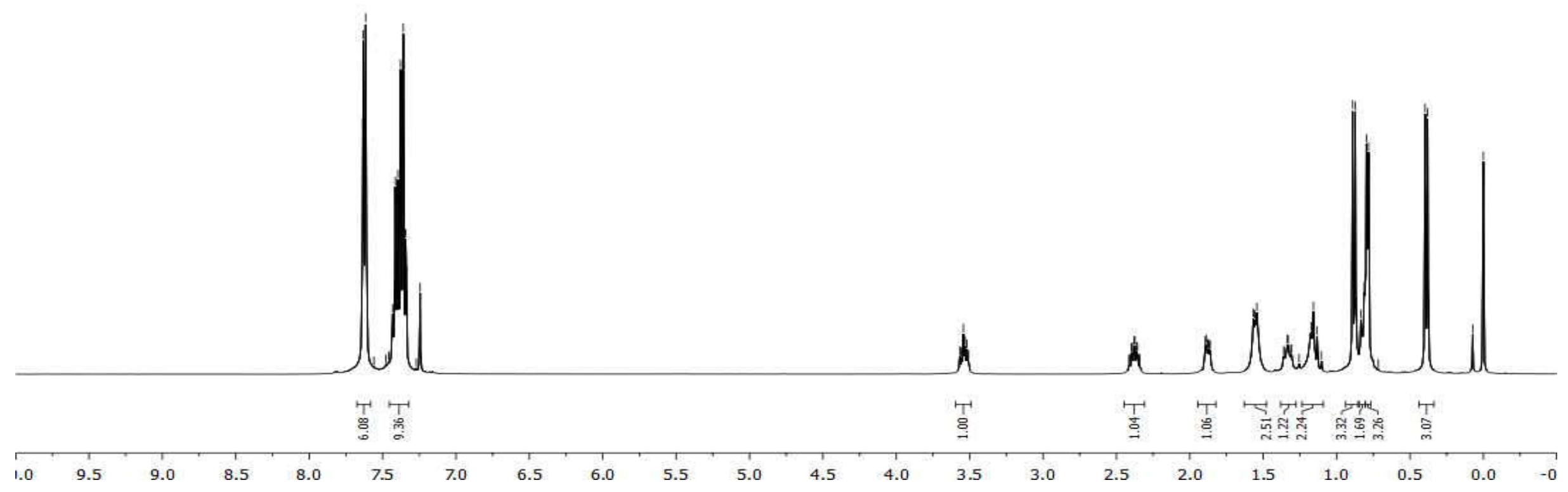




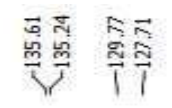

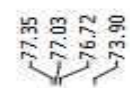

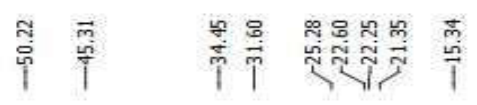

$\mathrm{OSiPh}_{3}$

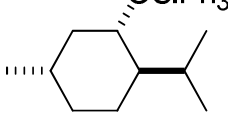

(D)-4

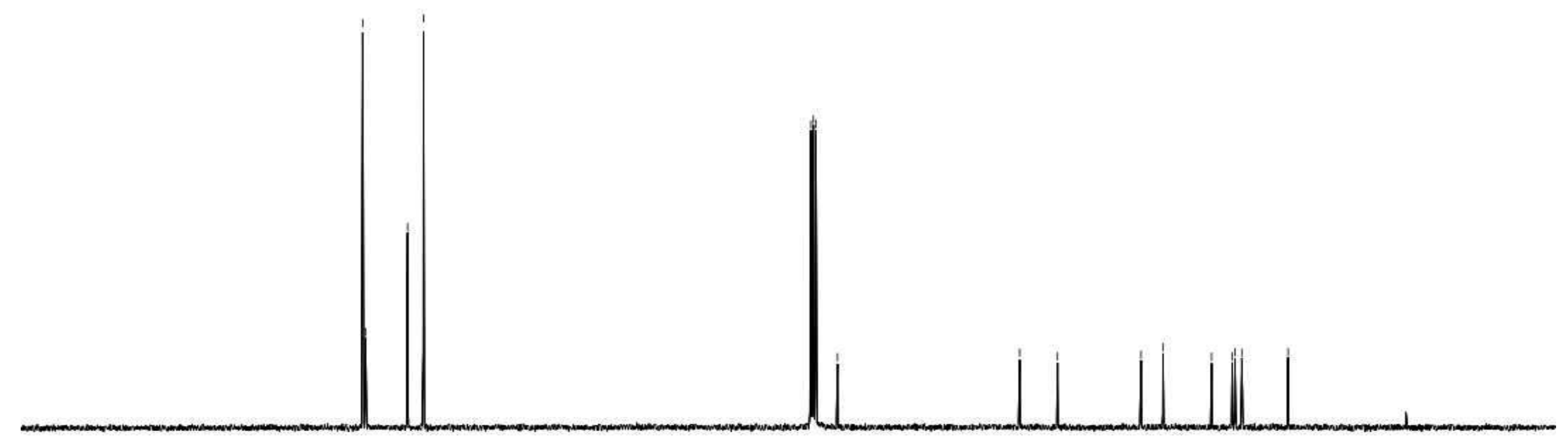




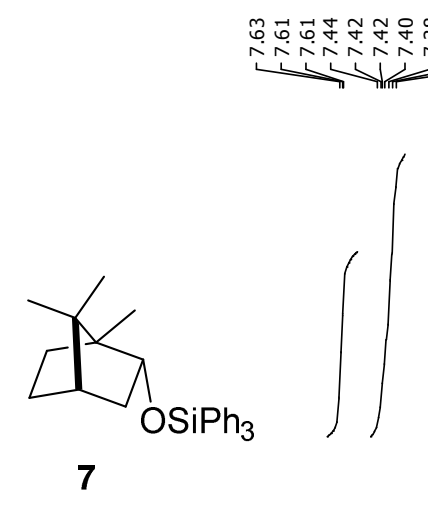

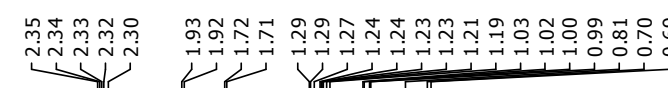

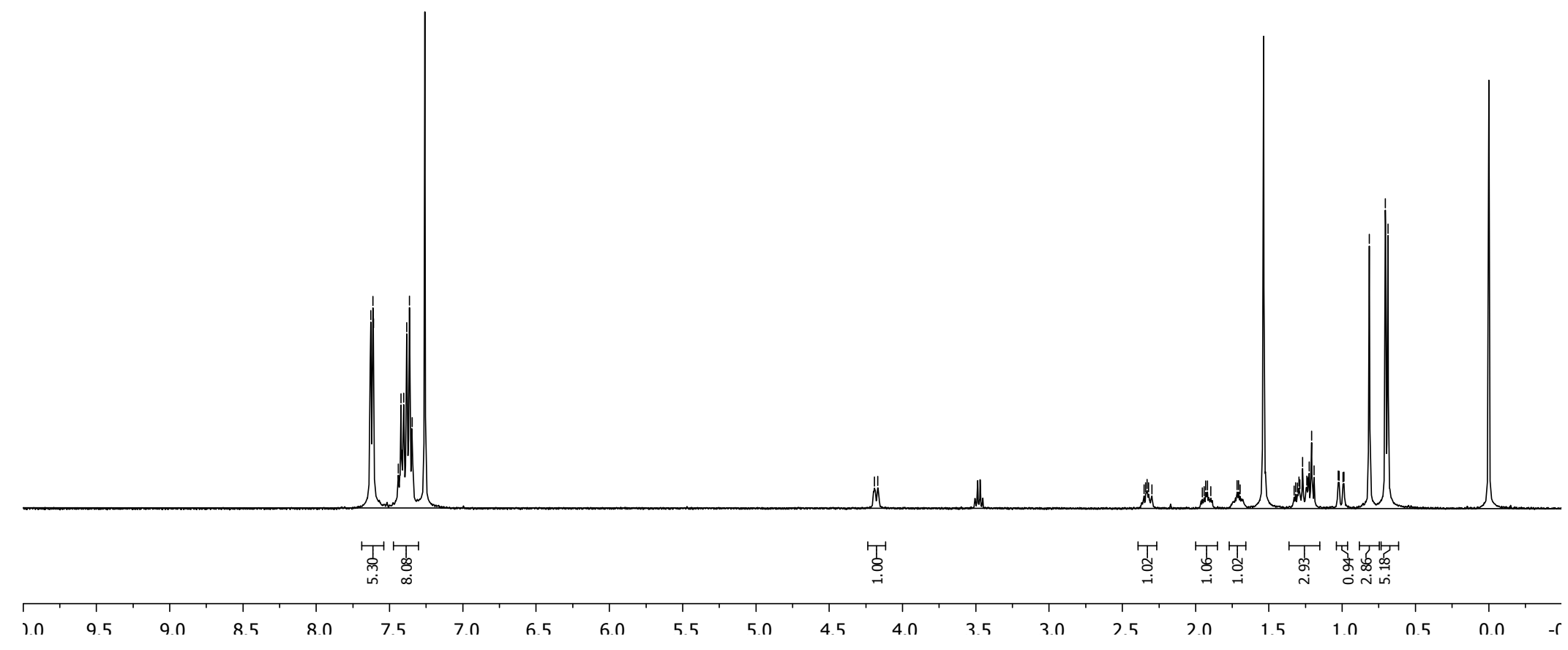



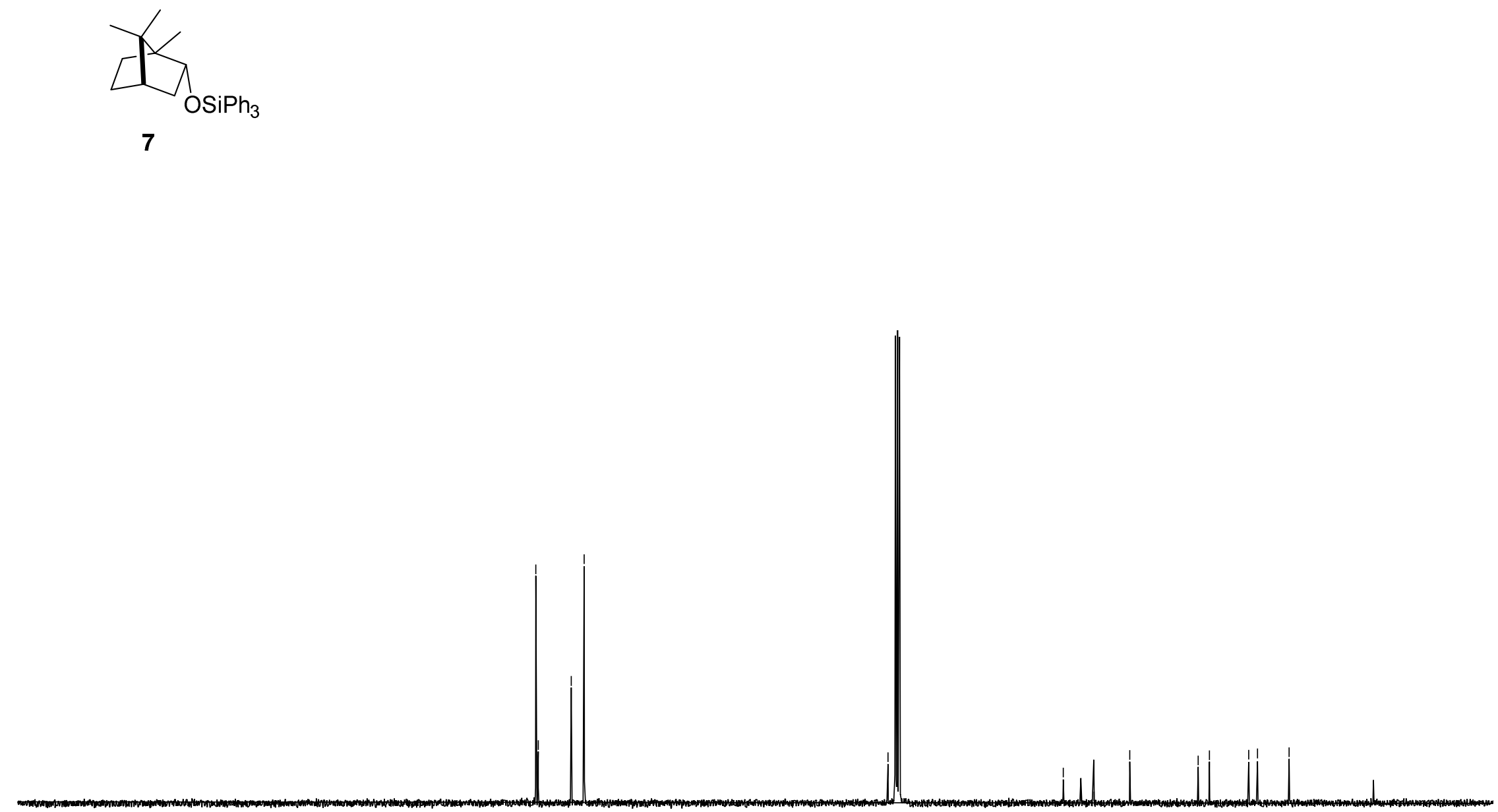

$210 \quad 2 n$

190

170 


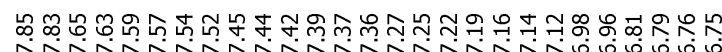

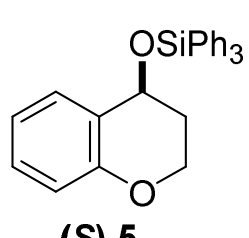

(S)-5

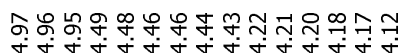

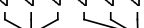

s s

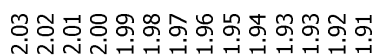

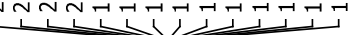

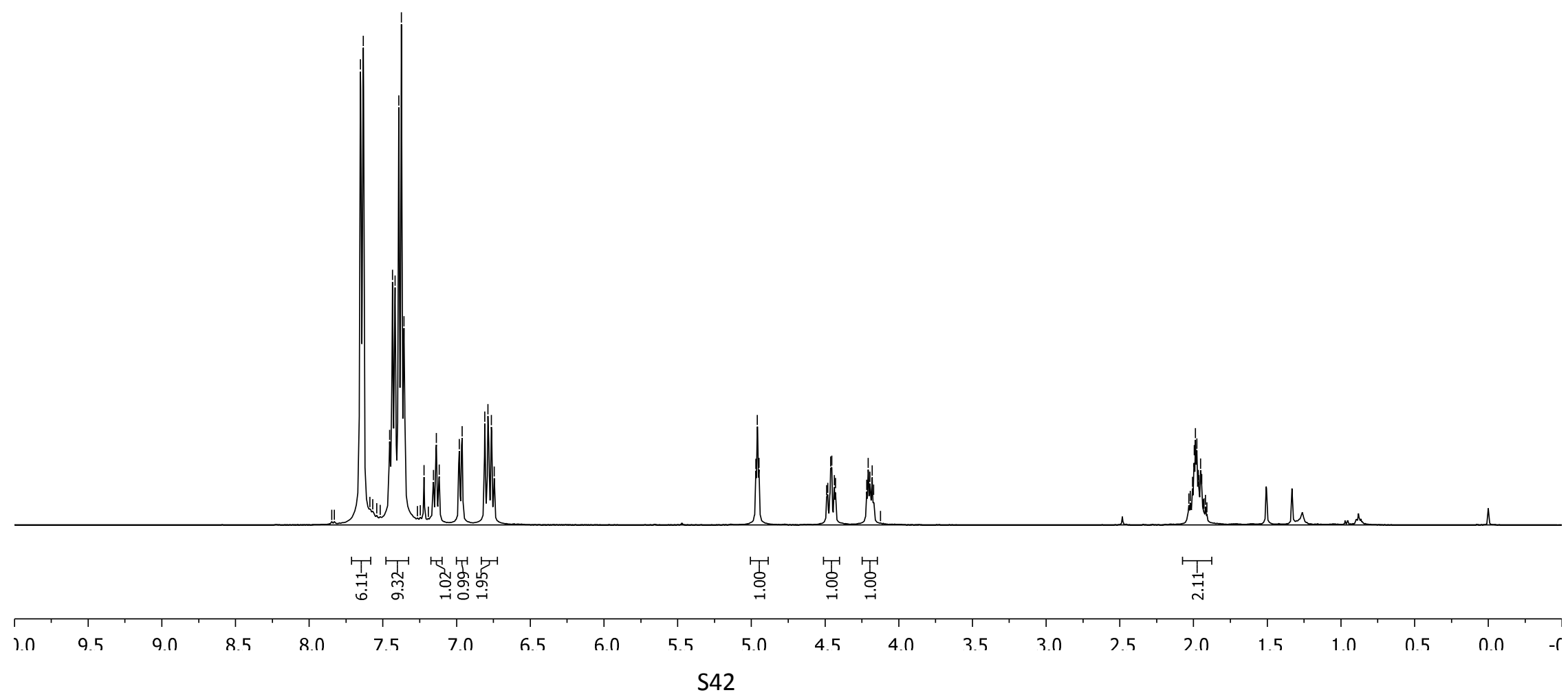



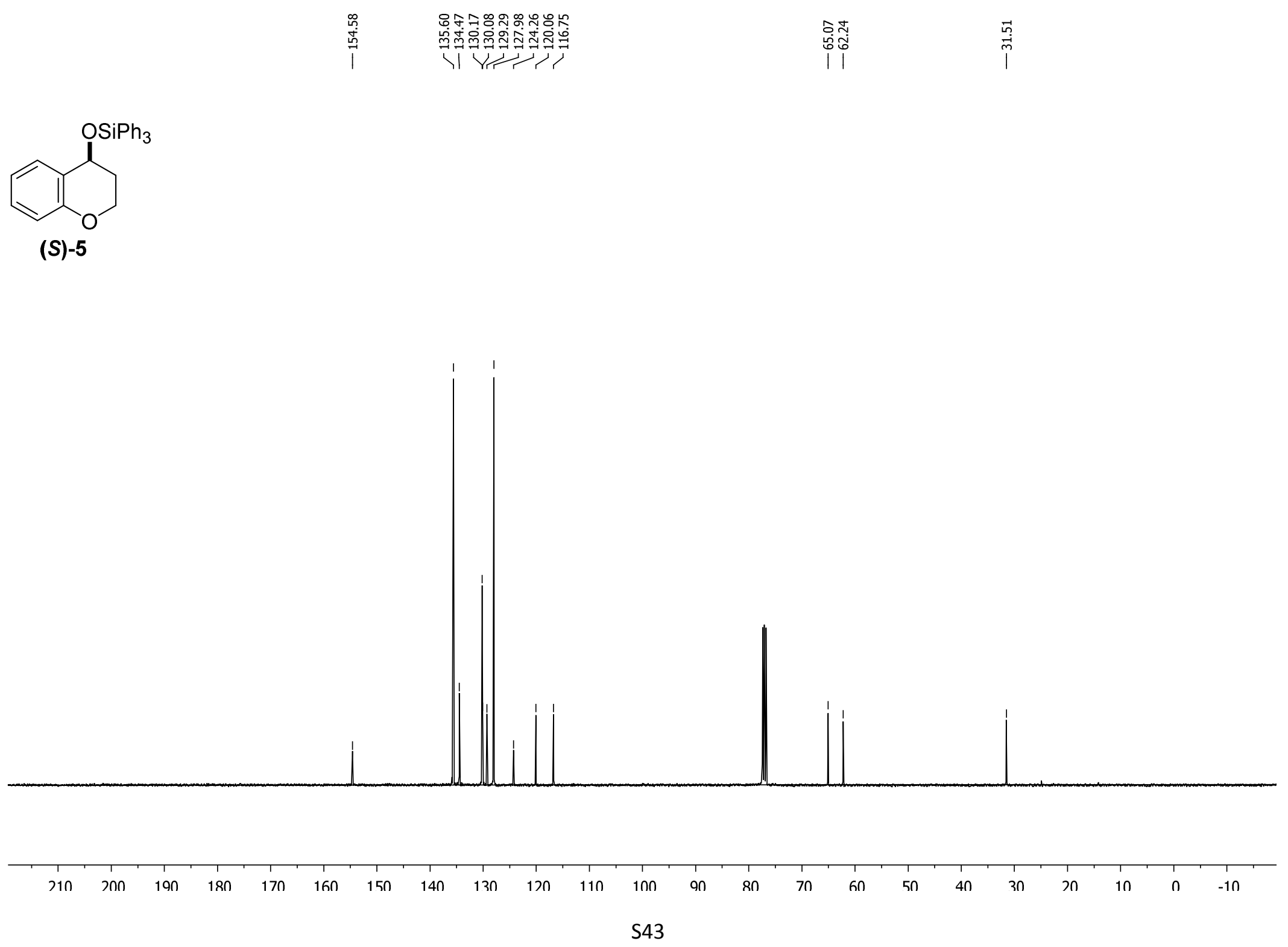


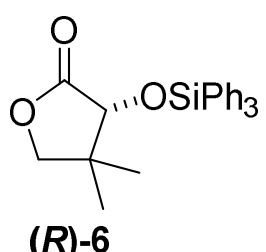

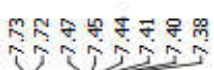

누ㅇㅛㅛ

$\stackrel{\circ}{i} \stackrel{0}{i}$

(R)-6

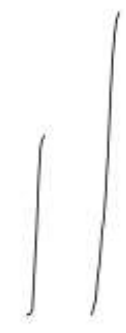

111
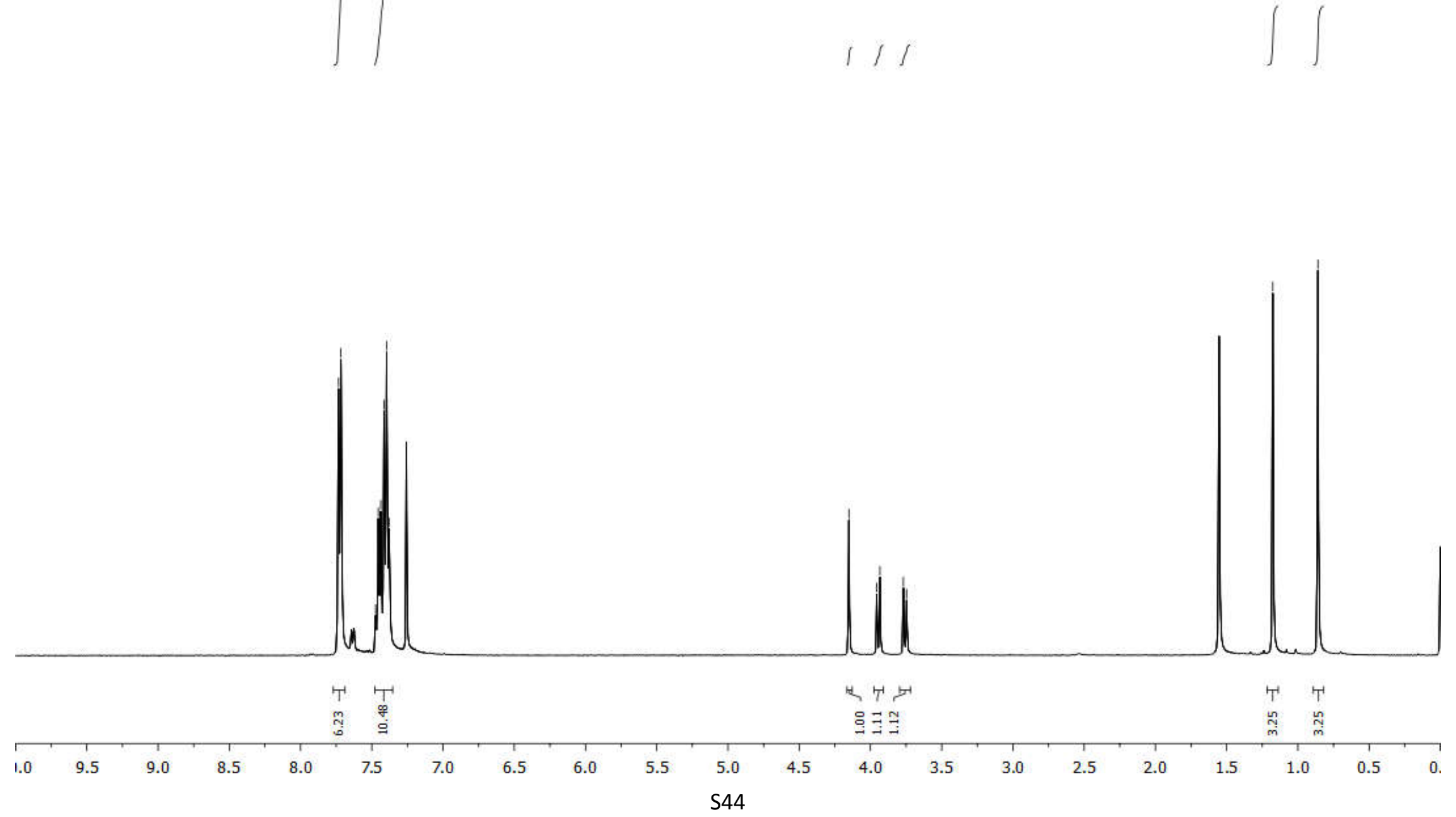


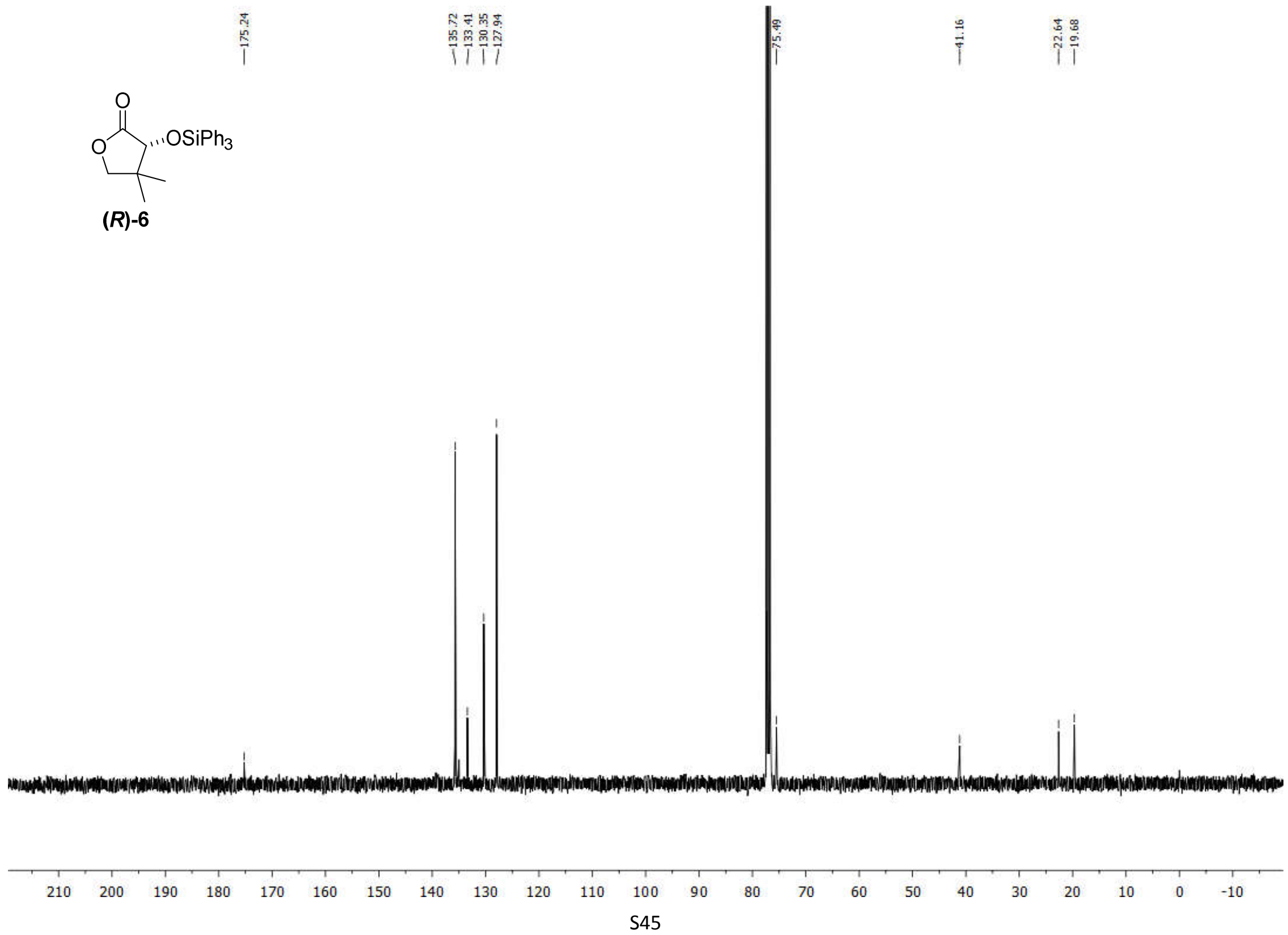




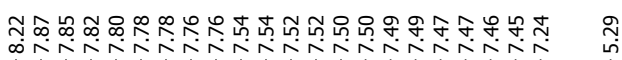

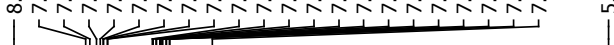
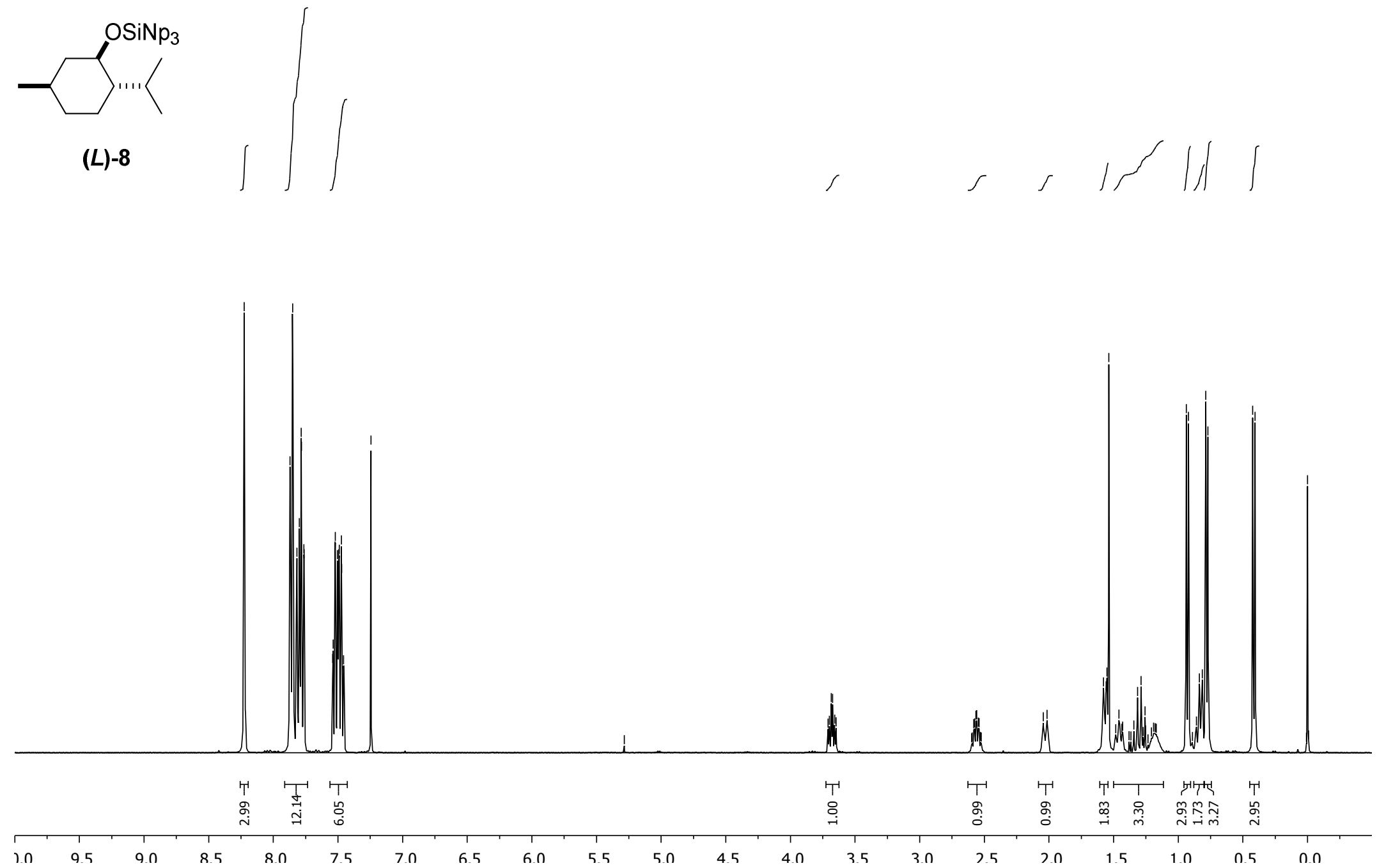

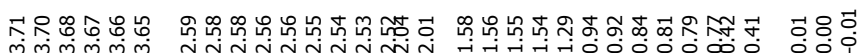

证

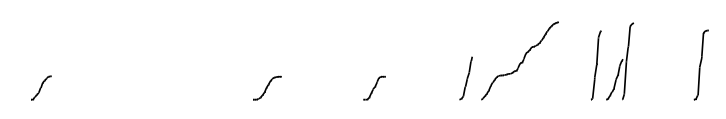



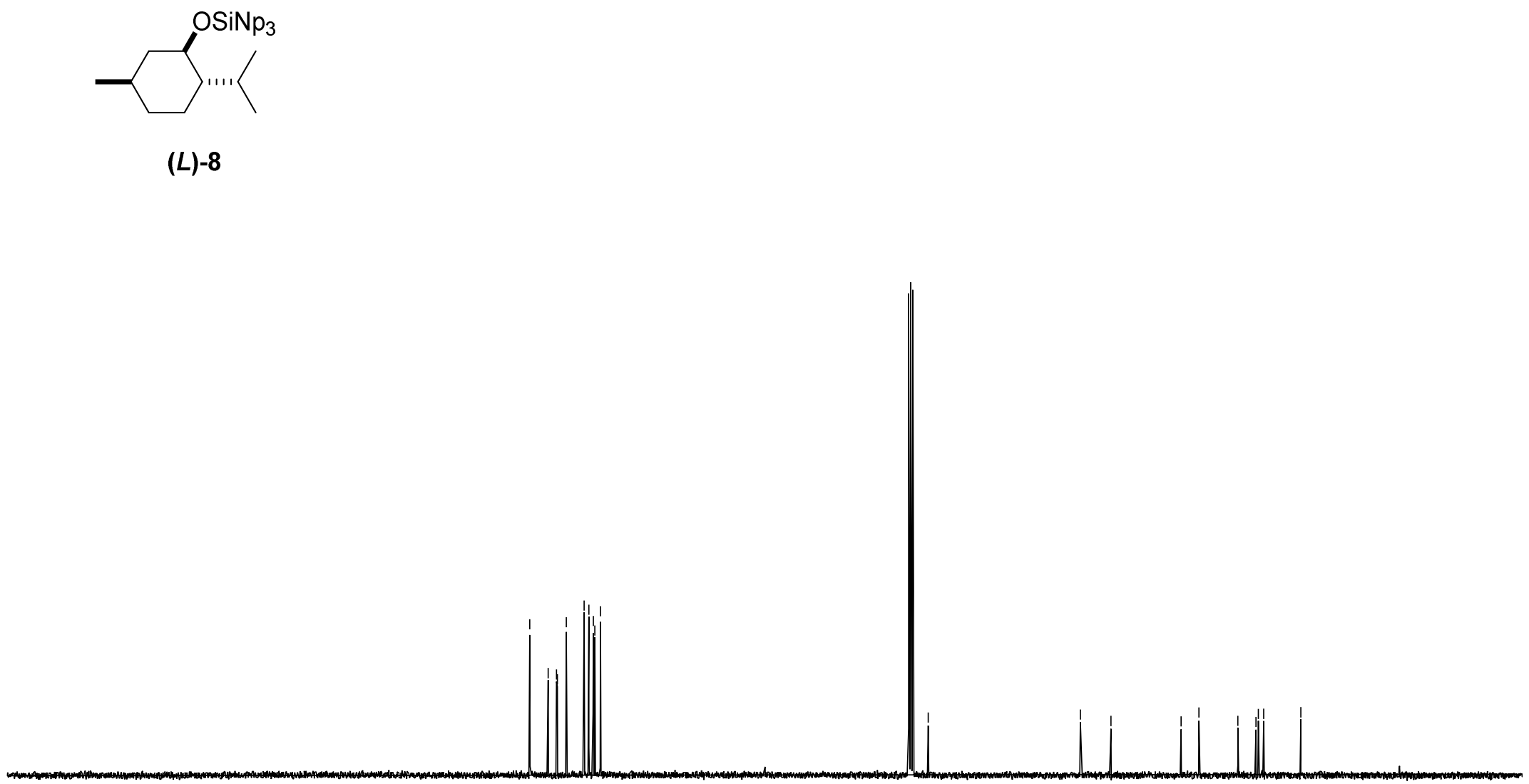
管

|

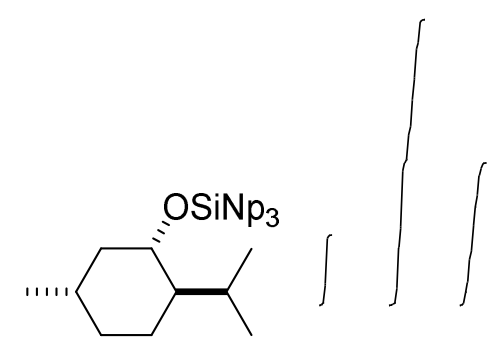

(D)-8

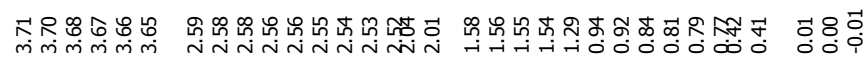

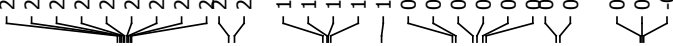

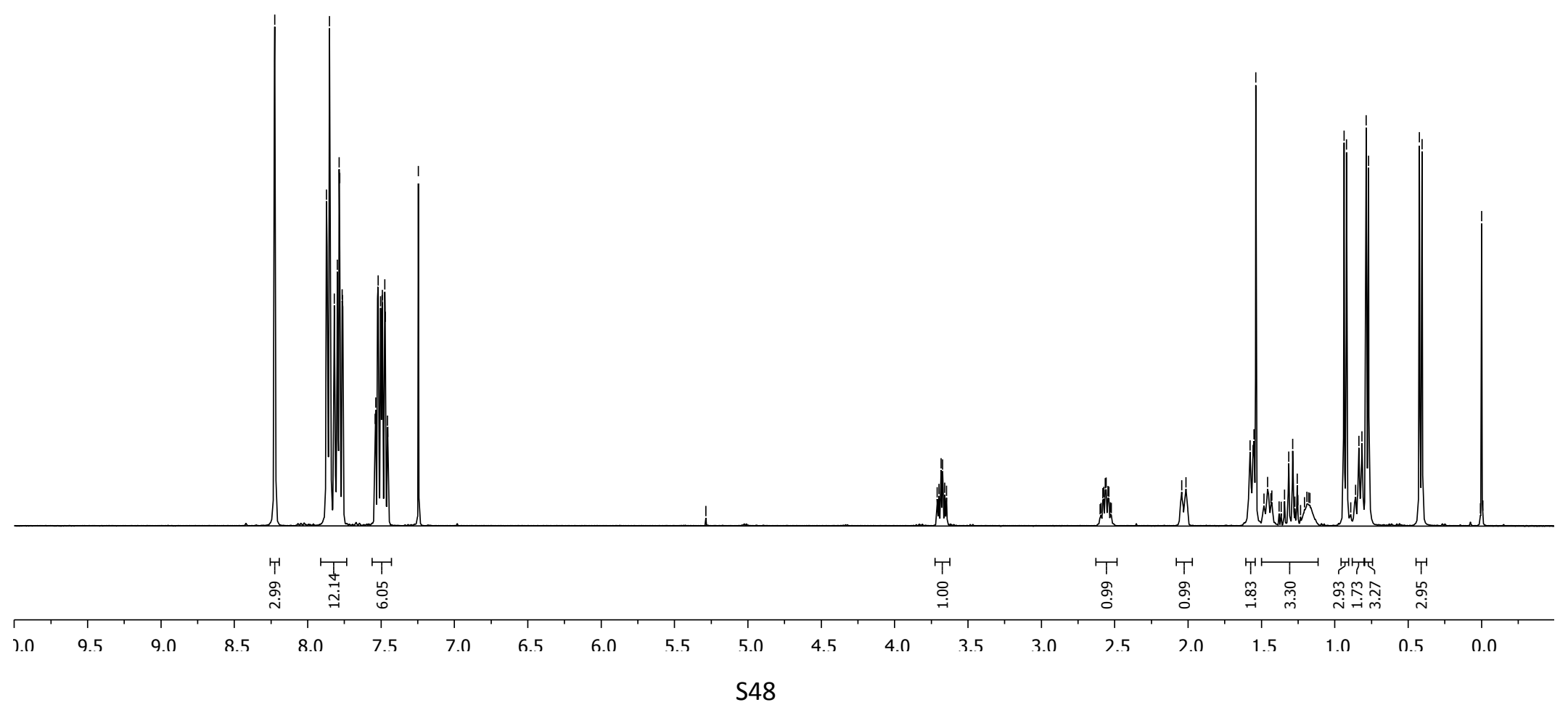



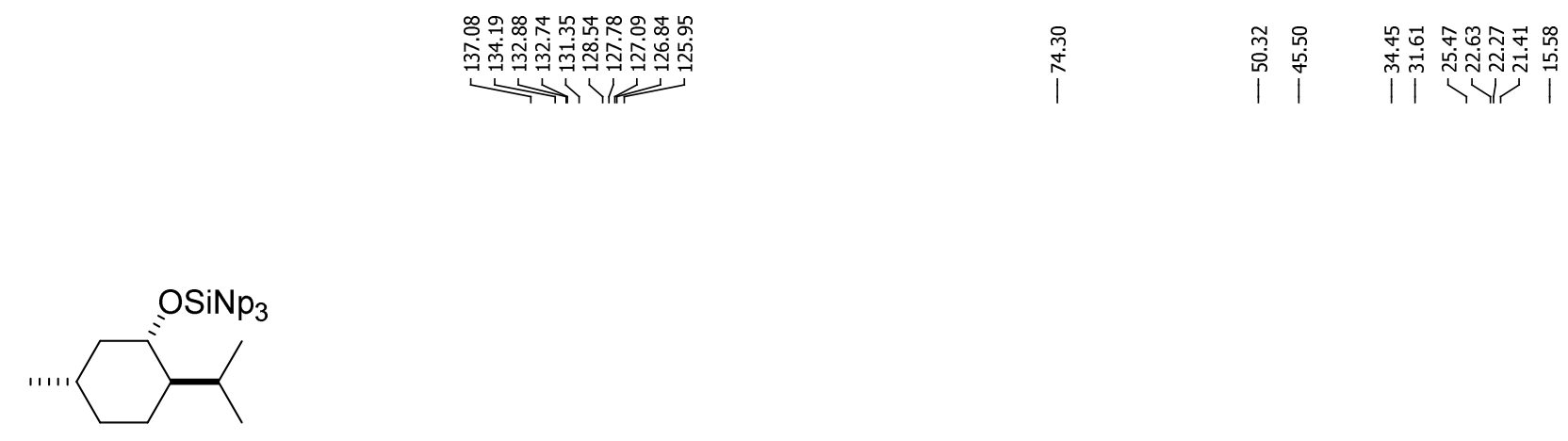

(D)-8

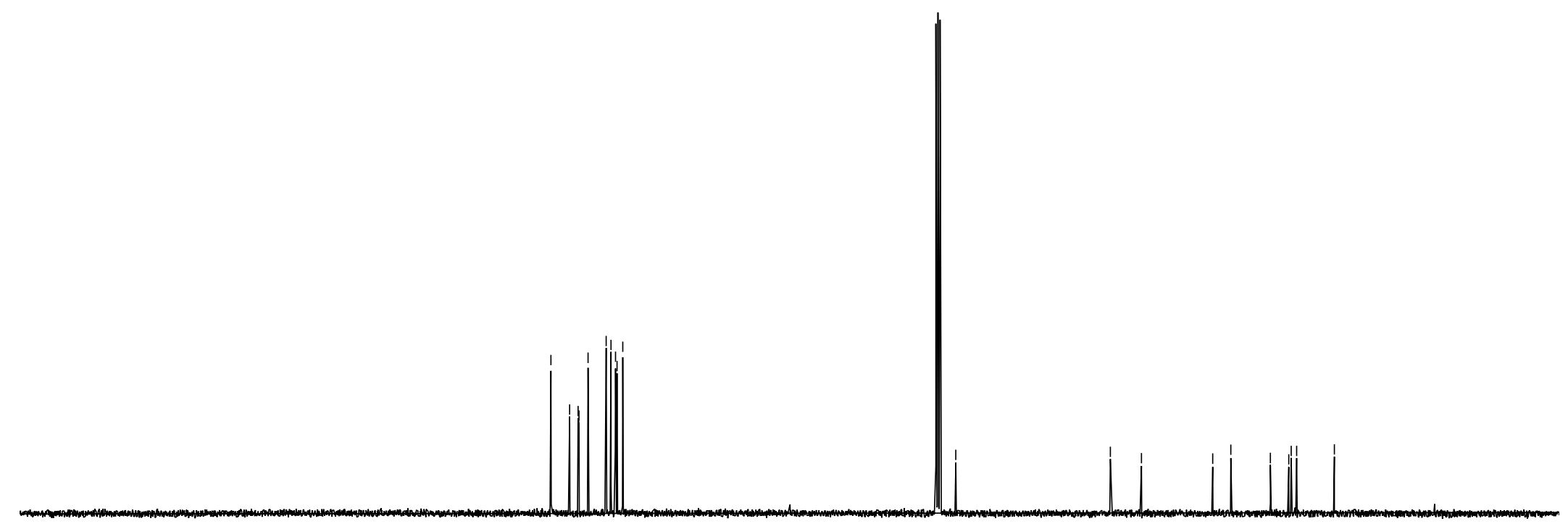

$210 \quad 200 \quad 190 \quad 180$

$150 \quad 140 \quad 130$

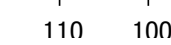

90 


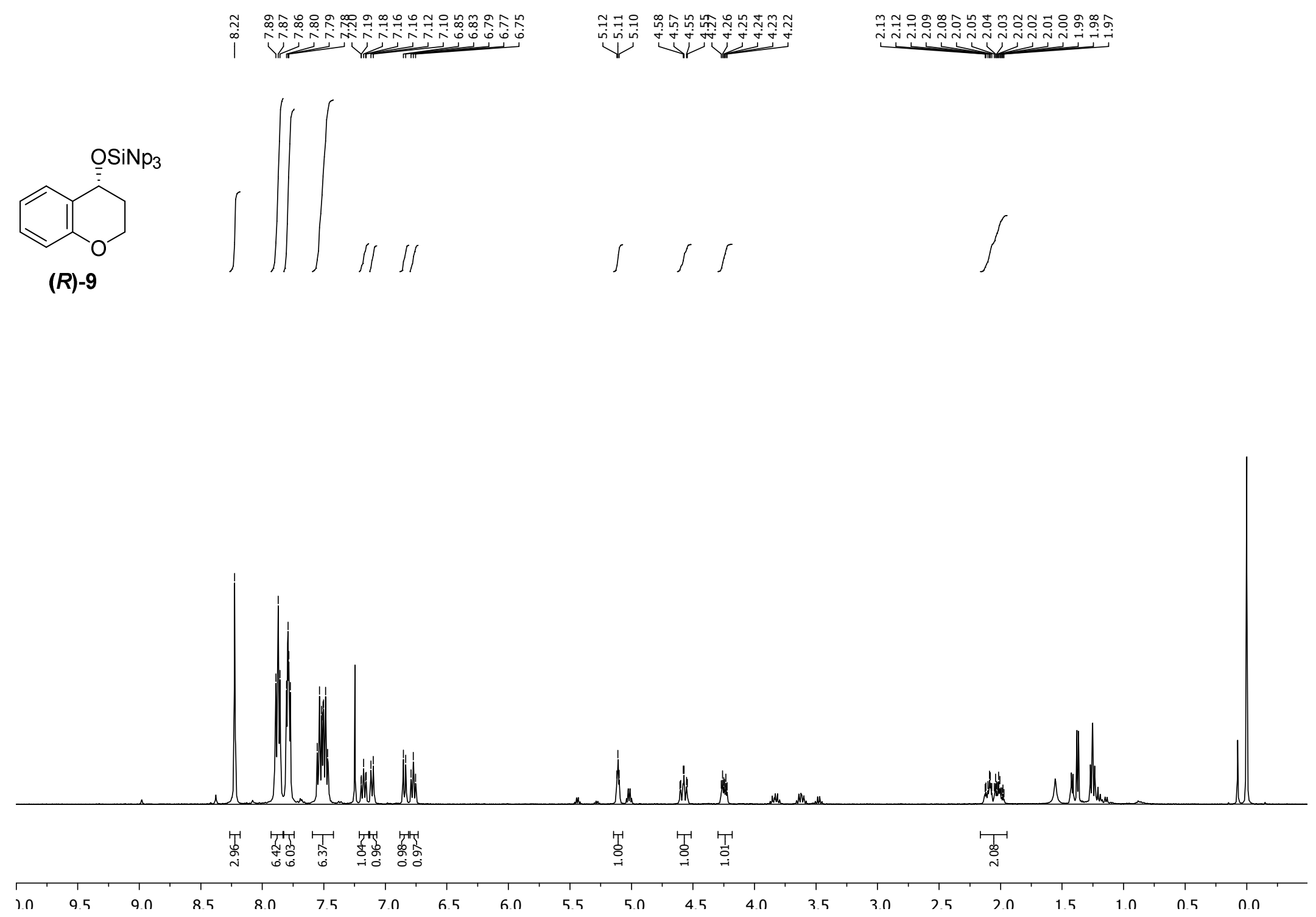




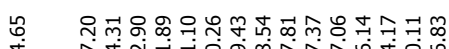

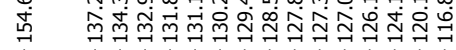

$1+2$

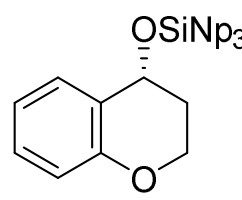

(R)-9
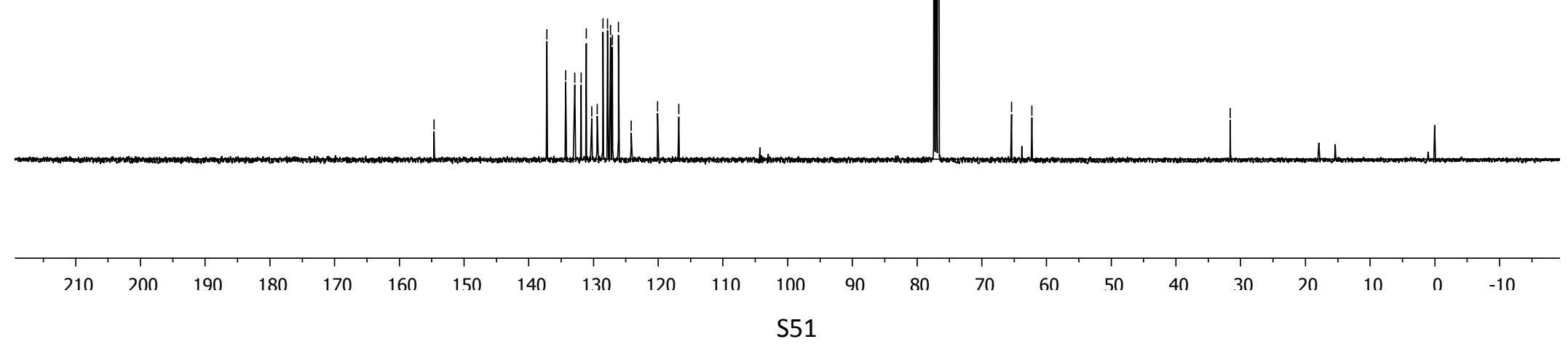


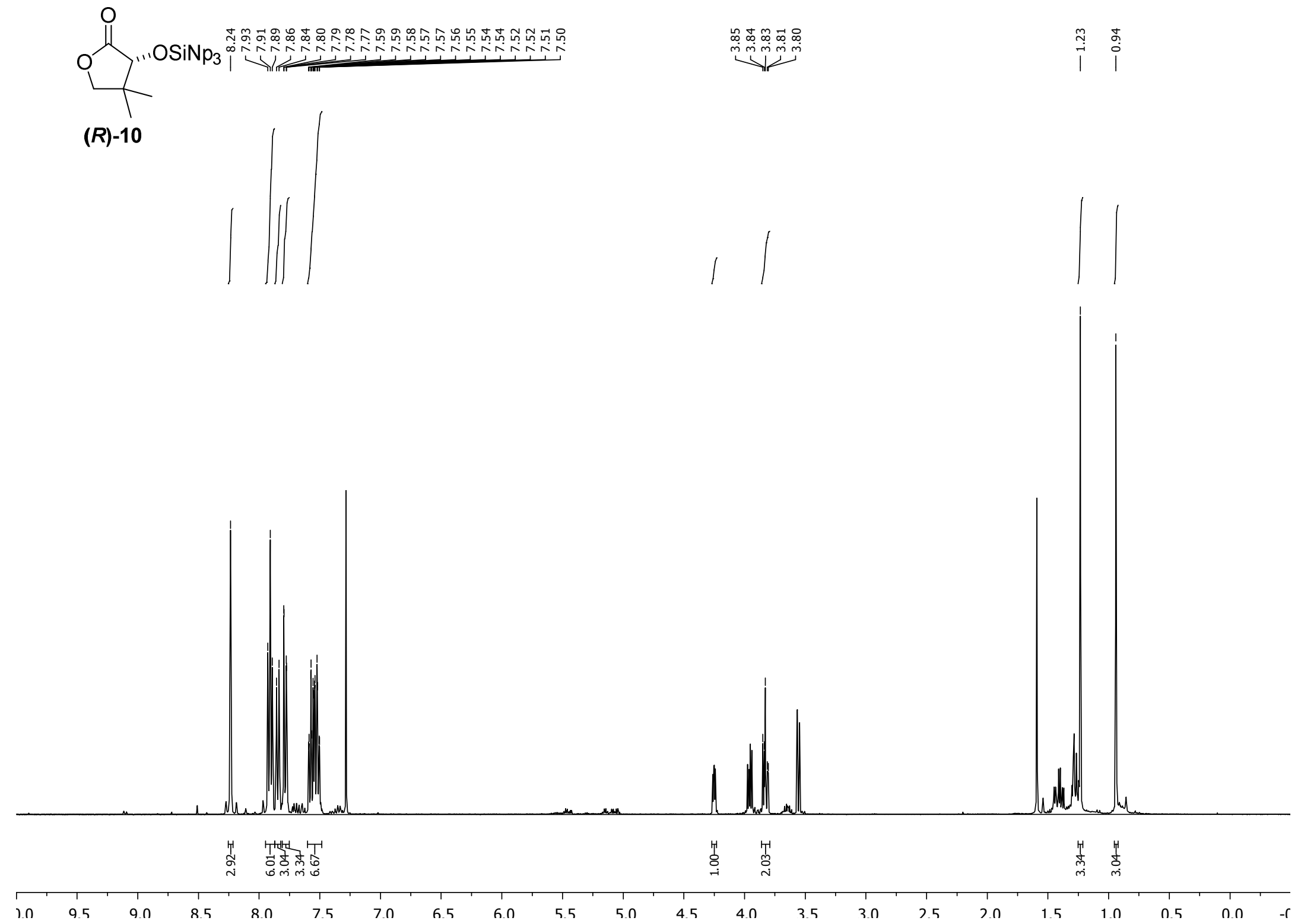



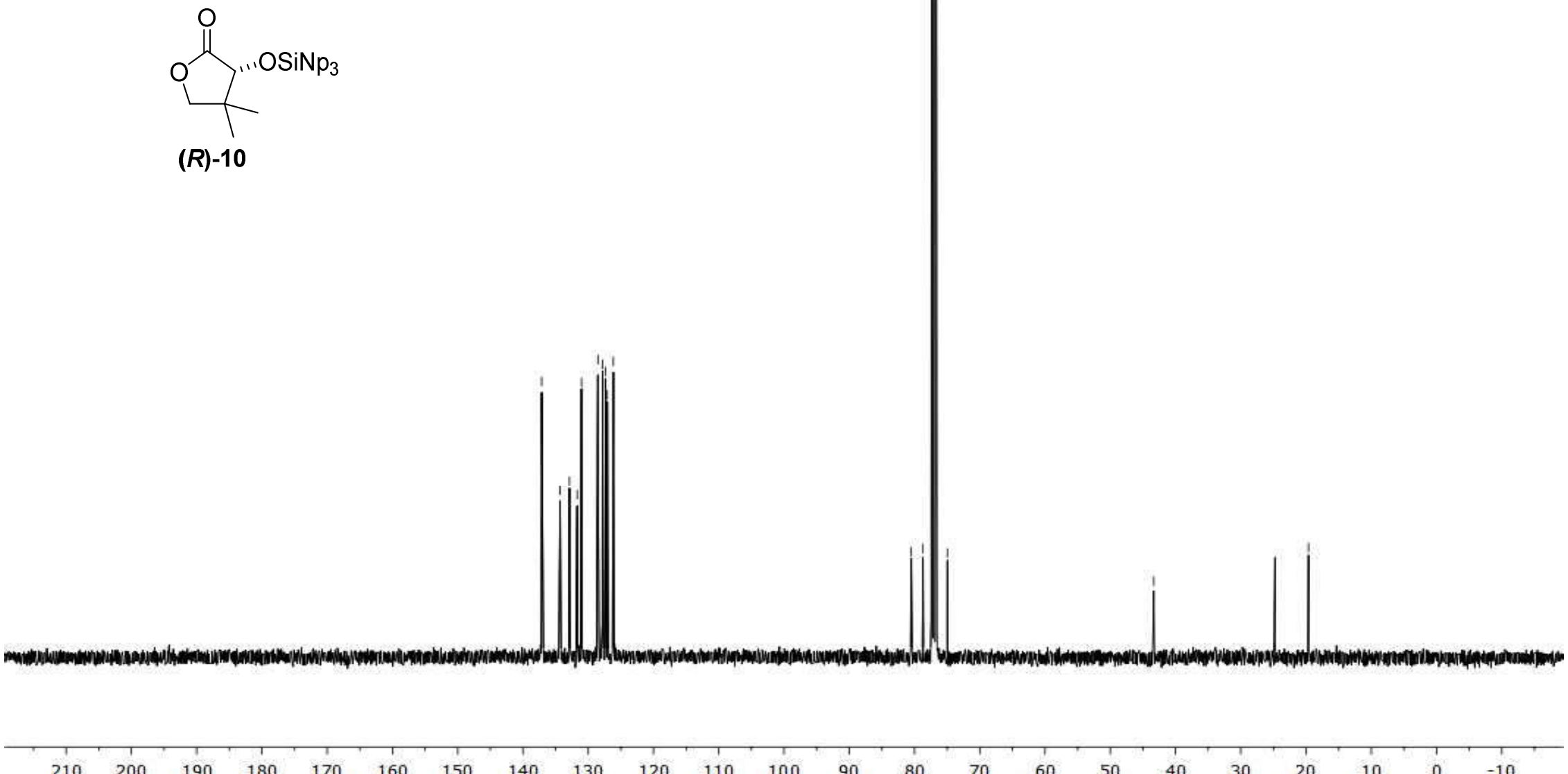

$\begin{array}{llll}160 & 150 & 140 & 130\end{array}$

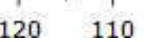

100.

90.

$80 \quad 70$

60

$50 \quad 40$

30

20

10

0 -10 